International Journal of Advancement in Life Sciences Research

Online ISSN: 2581-4877

journal homepage http://ijalsr.org

Original Article

\title{
A Taxonomic Account of Hover Flies (Insecta: Diptera: Syrphidae) with 2 New Records from Mid Hill Zone of Himachal Pradesh, India
}

\author{
Jayita Sengupta $^{1 \star}$, Atanu Naskar ${ }^{2}$, Sumit Homechaudhuri ${ }^{3}$, Dhriti Banerjee ${ }^{4}$ \\ ${ }^{1}$ Senior Zoological Assistant, Diptera Section, Zoological Survey of India, Kolkata, India \\ ${ }^{2}$ Assistant Zoologist, Diptera Section, Zoological Survey of India, Kolkata, India. \\ ${ }^{3}$ Professor, Department of Zoology, University of Calcutta, Kolkata, India. \\ ${ }^{4}$ Scientist-E, Diptera Section, Zoological Survey of India, Kolkata, India.
}

*Correspondence E-mail : jayitasengupta9@gmail.com*, atanudiptera@gmail.com, sumithomechaudhuri@gmail.com,dhritibanerjee@gmail.com.

\begin{abstract}
38 species of hover flies (Insecta: Diptera: Syrphidae) under 22 genera and 2 subfamilies has been reported from the Mid hill zone of Himachal Pradesh which includes a long stretch of elevational range varying from 651 to 1800 meters. 2 species namely Scavea pyrastri (Linnaeus, 1758) and Eristalinus (Eristalinus) polychromata (Fabricius, 1787) are reported for the first time from this Mid Hill zone as well as from the state of Himachal Pradesh. Their taxonomic keys and detail diagnosis of the reported species has been discussed along with the distributional pattern of these species along the Mid Hill Zone of Himachal Pradesh, India.
\end{abstract}

Keywords: Taxonomy, Hover flies, Syrphidae, New Record, Mid Hill zone, Himachal Pradesh.

\section{Introduction}

With a wide global distributional range and much wider list of known species $(6,000$ species under 300 groups approximately) Hover flies (Insecta: Diptera: Syrphidae) are one of the largest member of pollination network worldwide (Evenhuis \& Pape 2019). With a series of variability in terms of body sizes, habitat preferences, feeding habits, larval morphology, mimicry patterns (Thompson \& Vockeroth, 1982), almost all adult syrphids are identified based upon the the presence of spurious vein or false vein between the $3^{\text {rd }}$ and $4^{\text {th }}$ vein of the wing (Vockeroth, 1992).

Syrphidae being one of the predominant insects group in higher elevational landscape especially in mountainous ecosystem has always proved their efficiency as emerging leader of pollination (Ssymank et al, 2008). This fact is also supported by the hypothesis that the first liner hymenopterans tend to lose their pollination proficiency after a particular elevational range (Potts et al, 2013; Joshi \& Bhat, 2015), this fact is eventually expected to be worsen in future days with changing climatic conditions globally. (Lebuhn et al, 2013). These indicating towards a crisis in pollination network in higher elevational landscape in upcoming future eventually leading to a food crisis globally (Norman et al, 2008). Thus it has become urgent to develop a better knowledge of the pollinators in such eco system. Our present study area thus expanding through an wide elevational range of from $651 \mathrm{mt}(2,136 \mathrm{feet})$ to the range of 
$1800 \mathrm{mt}(5,906$ feet $)$ from the western Himalayan landscape of Himachal Pradesh. This elevation zone is more commonly categorised as the Mid hill zone based upon their agro climatic parameters. In the present study a brief synopsis on the taxonomy of the family is given along with its distributional pattern in the mid hill zone of Himachal Pradesh.

\section{Material and Methods}

\section{A. Study area:}

According to the Department of Agriculture, Himachal Pradesh the agro climatic condition has divided the whole state of Himachal Pradesh into 4 zones namely Shivalik hill zones, mid hill zones, high hill zone and cold and dry zone. Our present work is associated with the study areas from mid hill zones of
Himachal Pradesh. Our current study area includes the portion from Sirmour, Solan, Shimla, Mandi, Kullu, Kangra and of Pangi range of Chamba districts. Elevation of this zone ranges in approximately from $651 \mathrm{mt}$ to the 1,800 meters range of greater Himalaya in this state. This zone comprises about $32 \%$ of total geographical area of the State and 37\% of the total cultivated area of the total state. Such higher percentage of cultivated land from this region indicates towards its higher importance in term of agricultural values. The sub humid climatic condition of this area has accelerated the percentile of agricultural contribution from this agro climatic zone of Himachal Pradesh. Further this zone has very good potential in terms of production of both cash crops and temperate vegetables.

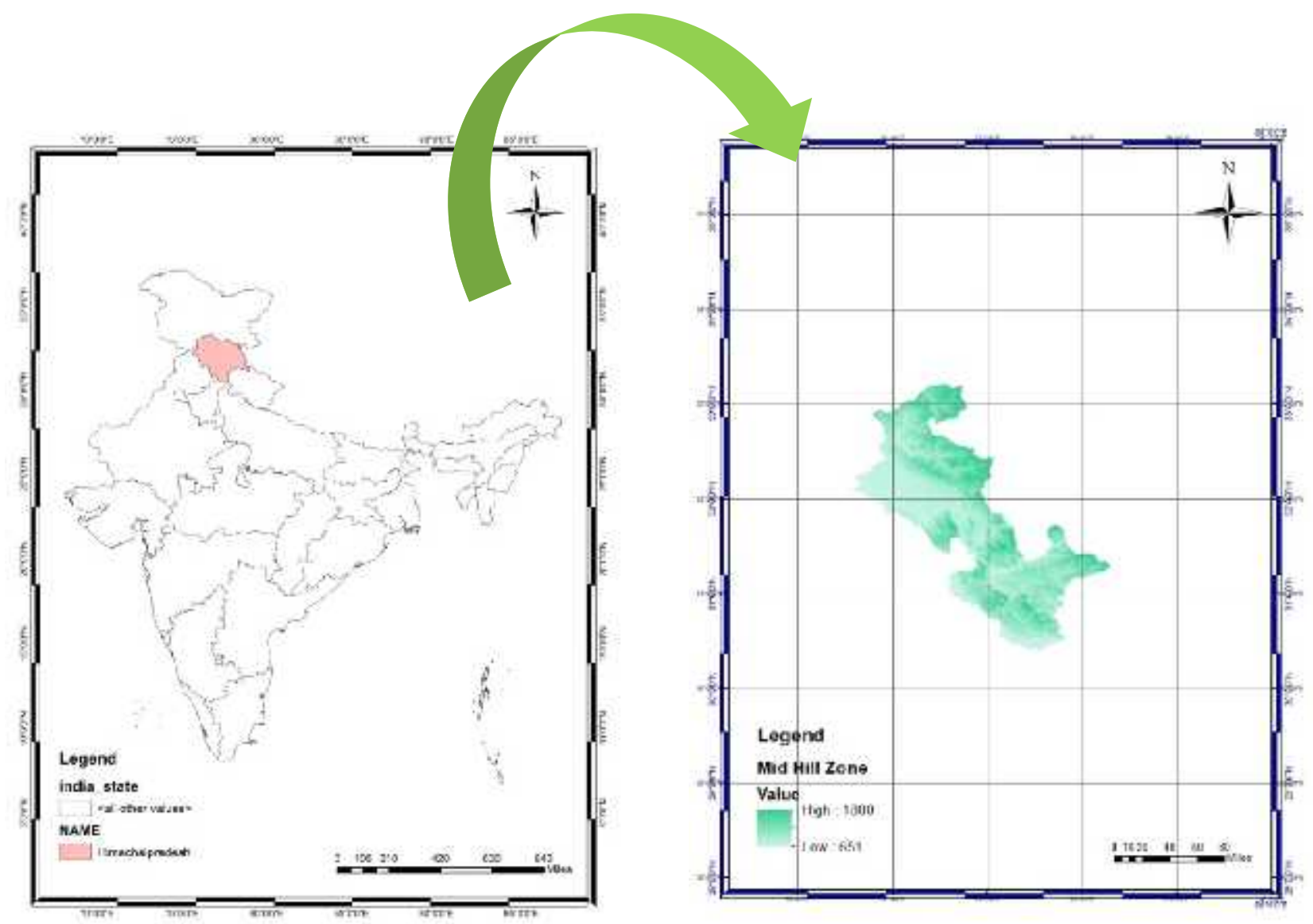

Figure 1A-1B: 3D Map showing 1A: State of Himachal Pradesh, 1B: Mid Hill zone as a part of Agro climatic zonation of the state. 


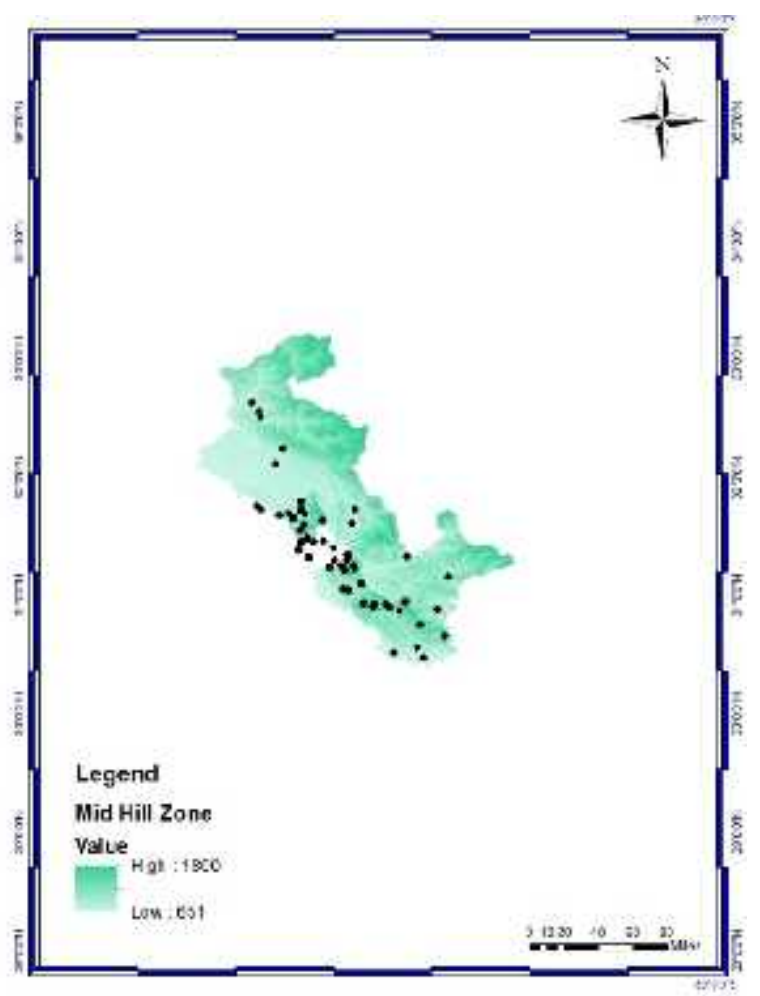

Figure 2: DEM Map showing species richness from mid hill zone from the state of Himachal Pradesh.

\section{B. Collection method:}

For the purpose of collection of hover flies, a 2 year long survey (2018-2019) has been conducted in this mid hill zone of Himachal Pradesh. Hoverflies were collected from the field during day time by using insect sweep nets, different type of traps like malaise trap, pan trap and UV light traps were used for collecting syrphid fauna. The collected samples are narcotized by using ethyl acetate and stored for further study in insect envelopes in the field. The specimens were later carried back to the laboratory, mounted on insect pins, labelled using the collection site information and stored in insect cabinets for further identification.

\section{Identification of specimens:}

Identification of the adults was done by following the keys of Teskey (1982), Miranda (2013), Vockeroth (1992) and Brunetti $(1907,1908,1913,1923)$ keeping in mind the recent nomenclatural changes (Pape and Thompson, 2018).Same has been used to construct taxonomic keys. All terminology while describing morphology has followed the recent pattern (Vockeroth et al. 1987).All the identified specimens were deposited in the designated repository of National Zoological Collection, Diptera section, Zoological Survey of India, Kolkata.

\section{Technical procedure:}

The Digital Elevation Model (DEM) map of study area is generated by using ARC GIS software version 10.1. Microsoft Excel version 2013 has been used here for generation of graphs.

\section{Results and Discussion}

Altogether 38 species of hoverflies under 22 genera and 2 sub families have been reported from our study area. Among which 2 species namely 2 species namely Scavea pyrastri (Linnaeus, 1758) and Eristalinus (Eristalinus) polychromata (Fabricius, 1787) are reported for the first time from this Mid Hill zone as well as from the state of Himachal Pradesh. Detailed systematic account along with taxonomic key has been discussed. Distribution pattern of all syrphid species has been discussed in detail.

\section{List of Taxa}

Suborder Brachycera Macquart, 1834

clade Eremoneura Lameere, 1906

clade Aschiza Becher, 1882

super family Syrphoidea Latreille, 1802

Family SYRPHIDAE Latreille, 1802

Subfamily SYRPHINAE Latreille, 1802

Tribe Syrphini Latreille, 1802

I. Genus Asarkina Macquart, 1842

Subgenus Asarkina Macquart, 1842

1. Asarkina(Asarkina) ericetorum (Fabricius, 1781)

II. Genus Dasysyrphus Enderlein, 1938

2. Dasysyrphus orsua (Walker, 1852)

III. Genus Episyrphus Matsumura \& Adachi, 1917

Subgenus Episyrphus Matsumura \& Adachi, 1917 
3. Episyrphus(Episyrphus)balteatus (De Geer, 1776)

IV. Genus Eupeodes OstenSacken, 1877

Subgenus Macrosyrphus Matsumura, 1917

4. Eupeodes (Macrosyrphus) confrater (Wiedemann, 1830)

Subgenus Metasyrphus Matsumura, 1917

5. Eupeodes(Metasyrphus)/atifasciatus (Macquart, 1829)

V. Genus Ischiodon Sack, 1913

6. Ischiodon scutellaris (Fabricius, 1805)

VI. Genus Meliscaeva Frey, 1946

7. Meliscaeva cinctella (Zetterstedt, 1843)

VII. Genus Scaeva Fabricius, 1805

8. Scaeva selenitica (Meigen, 1822)

9. Scaeva pyrastri (Linnaeus, 1758)

Subgenus Sphaerophoria Wiedemann, 1830

10. Sphaerophoria(Sphaerophoria) indiana Bigot, 1884

Subgenus Knutsonia Barkalov, 2012

11. Sphaerophoria viridaeneaBrunetti, 1915

VIII. Genus Syrphus Fabricius, 1775

Subgenus Syrphus Fabricius, 1775

12. Syrphus(Syrphus) fulvifacies Brunetti, 1913

IX. Genus Chrysotoxum Meigen, 1800

13. Chrysotoxum convexam Brunetti, 1915

14. Chrysotoxum violaceum Brunetti, 1923

Tribe Bacchini Bigot, 1883

X. Genus Baccha Fabricius, 1805

15. Baccha maculata Walker, 1852

XI. Genus Melanostoma Schiner, 1860
16. Melanostoma orientale (Wiedemann, 1824)

Tribe Paragini Glumac, 1961

XII. Genus Paragus Latreille, 1804

Subgenus Paragus Latreille, 1804

17. Paragus(Paragus)serratus (Fabricius, 1805)

Subfamily ERISTALINAE

Tribe Rhingiini

XIII. Genus Cheilosia Meigen, 1822

18. Cheilosia nigroaenea Brunetti, 1915

Tribe Volucellini Newman, 1834

XIV. Genus Volucella Geoffroy, 1762

19. Volucella ruficauda Brunetti, 1907

XV. Genus Sphiximorpha Rondani, 1850

20. Sphiximorpha triangulifera (Brunetti, 1913)

Tribe Eristalini Newman, 1834

XVI. Genus Eristalinus Rondani, 1845

21. Eristalinus (Eristalinus) arvorum (Fabricius, 1787)

22. Eristalinus (Eristalinus) megacephalus (Rossi,1794)

23. Eristalinus (Eristalinus) polychromata

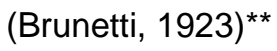

24. Eristalinus (Eristalinus) tabanoides (Jaennicke, 1867)

25. Eristalinus(Eristalinus) quinquestriatus (Fabricius, 1794)

Subgenus Eristalodes Mik, 1897

26. Eristalinus (Eristalodes)taeniops (Wiedemann, 1818)

27. Eristalinus (Eristalodes) paria (Bigot, 1880)

XVII. Genus Eristalis Latreille, 1804

Subgenus Eoseristalis Kanervo, 1938

28. Eristalis(Eoseristalis) albibasis Bigot, 1880 
29. Eristalis (Eoseristalis) arbustorum (Linnaeus, 1758)

30. Eristalis(Eoseristalis) cerealisFabricius , 1805

31. Eristalis(Eoseristalis) himalayensisBrunetti, 1908

Subgenus Eristalis Latreille, 1804

32. Eristalis (Eristalis) tenax (Linnaeus, 1758)

XVIII. Genus Mallota Meigen, 1822

Subgenus Mallota Meigen, 1822

33. Mallota (Mallota) orientalis Wiedemann, 1824

XIX. Genus Mesembrius Rondani, 1857

Subgenus Mesembrius Rondani, 1857

34. Mesembrius

(Mesembrius) bengalensis (Wiedemann, 1819)

XX. Genus Lycastris Walker, 1857

35. Lycastris flavohirta Brunetti, 1907

XXI. Genus Syritta Lepeletier \& Serville, 1828

36. Syritta indica (Wiedemann, 1824)

37. Syritta pipiens (Linnaeus, 1758)

XXII. Genus Xylota Meigen, 1822

Subgenus Xylota Meigen, 1822

38. Xylota(Xylota) nursei Brunetti, 1923

**: new record from state

Family Syrphidae (New Records from the state of Himachal Pradesh has been demarcated with asterisk)

\section{Systematic account}

Tribe Syrphini Latreille, 1802

I. Genus Asarkina Macquart, 1842

Subgenus Asarkina Macquart, 1842

1. Asarkina(Asarkina)ericetorum (Fabricius, 1781)

1781. Syrphus ericetorum Fabricius, Spec. Insect. ,2: 425.

Type-locality: Africa aequinoctial.
Material examined: $10^{\pi}$ Shilwara, Sirmour district, $1292 \mathrm{mt}$, 3044'56.43"N,77²9'51.14"E, 16.iv.17, coll:J.

Sengupta, $20^{x} 0^{x} 2+9$ Jagatpur, Sirmour district, $900 \mathrm{mt}, 30^{\circ} 28^{\prime} 13.55^{\prime \prime} \mathrm{N}, 7^{\circ} 31^{\prime} 22.30^{\prime \prime} \mathrm{E}$, 16.iv.17, coll: J. Sengupta, $10^{\top}$ Dewni, Sirmour district, $900 \mathrm{mt}, 30^{\circ} 30^{\prime} 43.49^{\prime \prime N}$, 77¹6'9.74"E, 16.iv.17, coll: J. Sengupta,1우 Chakmoh Berthin roadside, Hamirpur district, $842 \mathrm{mt}$, 31²6'49.71"N, 76³5'17.87"E, 03.ii.16, coll: J. Sengupta, 2 우 우 Chabutar village, Hamirpur district, $774 \mathrm{mt}$, 3141'10.2"N,76³1'16.7"E,02.ii.16,coll: J. Sengupta, 2 ㅇ Kanti Mishwa, Sirmour district, $1467 \quad \mathrm{mt}, \quad 30^{\circ} 36^{\prime} 43.78^{\prime \prime} \mathrm{N}$, 77³8'47.37"E,16.iv.17,coll:J. Sengupta, $10^{*}$ $\mathrm{Nei}$ Neti, Sirmour district, $1323 \mathrm{mt}$, 3053'53.17"N, 77¹4'14.05"E,16.iv.17,coll:J. Sengupta, $10^{\pi}$ Bhanat, Sirmour district, 1672 $\mathrm{mt}, \quad 30^{\circ} 52^{\prime} 2.62^{\prime \prime} \mathrm{N}$, 77019'14.00"E,16.iv.17, coll:J. Sengupta, $10^{*}$ Matiana, Sirmour district, $1184 \mathrm{mt}$, 30³9'13.49"N, 77²2'4.88"E,16.iv.17, coll:J. Sengupta, 2 ㅇ Batyana, Hamirpur district, $784 \mathrm{mt}, 31^{\circ} 29^{\prime} 4.49 " \mathrm{~N}, 76^{\circ} 31^{\prime} 50.78^{\prime \prime} \mathrm{E}, 03 . i i .16$, coll: J. Sengupta.

Distribution: Himachal Pradesh, Arunachal Pradesh, Assam, Chandigarh, Gujarat, Jammu \& Kashmir, Manipur, Meghalaya, Sikkim, Tamil Nadu, Tripura, Uttarakhand, Uttar Pradesh, West Bengal.

Elsewhere: Afrotropical Region (Africa), Australasian Region (Australia, New Guinea), Neo tropical Region (Formosa), Oriental Region (Nepal, Srilanka, S-E Asia).

\section{Genus Dasysyrphus Enderlein, 1938}

2. Dasysyrphus orsua (Walker, 1852)

1852. Syrphus orsua Walker, Insecta. Saund.1:231

Type-locality: East Indies.

Material examined: $1+20^{x} 0^{x}$ Renuka lake, Sirmour district, $672 \mathrm{mt}$, 32³3'12.1"N,76³'32.9"E, 15.iv.17, coll: J. Sengupta,2우우 Dadahu Sirmour district, 651 mt, 30³3'46.2456"N.77²8'12.7086"E16.iv.17, coll: J. Sengupta.

Distribution: India: Himachal Pradesh, Jammu \& Kashmir, Uttarakhand, West Bengal. 
Elsewhere: Oriental Region (Nepal, Sri Lanka, Sumatra.)

\section{Genus Episyrphus Matsumura \& Adachi, 1917}

Subgenus Episyrphus Matsumura \& Adachi, 1917

\section{Episyrphus(Episyrphus)balteatus (De Geer, 1776)}

1776. Musca balteata De Geer, Mem. Pour. serv. Hist. Ins. 6:116

Type locality: Sweden.

Material examined: $40^{x} 0^{x}$, Bhowen, Chamba district, $2200 \mathrm{mt} ; \quad 32^{\circ} 29^{\prime} 55.60^{\prime \prime} \mathrm{N}, \quad 76^{\circ}$ 5'27.40"E, 15.iv.17, coll: J.Sengupta, $60^{x} 0^{x}$, 3 우우 Renuka Lake, Sirmour district, 650mt, 32³3'12.1"N, 767'32.9"E, 15.iv.17, coll: J.Sengupta, $20^{\pi} \sigma^{\pi}$, Renuka Lake, Sirmour district, 672mt, 32॰33'12.1"N,, 76² $32.9^{\prime \prime} \mathrm{E}$, 15.iv.17, coll: J.Sengupta, $40^{\pi} 0^{\pi}$, 3 우우 Dadahu, Sirmour district, $651 \mathrm{mt}$, 30³3'46.2"N,77²8'12.7"E, 15.iv.17, coll: J.Sengupta, 3우우 $20^{x} 0^{x}$ Renuka lake, Sirmour district, $672 \mathrm{mt}, 32^{\circ} 33^{\prime} 12.1^{\prime \prime} \mathrm{N}, 7^{\circ} 7^{\prime} 32.9^{\prime \prime} \mathrm{E}$, 15.iv.17, coll: J. Sengupta,4우 우 Dadahu Sirmour district, $651 \mathrm{mt}$, 30³3'46.2456"N.77²8'12.7086"E16.iv.17, coll: J. Sengupta, $110^{\pi} 0^{\pi} 4$ 우 ㅇ B Bergaon, Solan district, $1452 \mathrm{mt}, 30^{\circ} 54^{\prime} 16.1^{\prime \prime} \mathrm{N}, 7^{\circ} 5^{\prime} 48.2^{\prime \prime} \mathrm{E}$, 18.iv.17, coll: J. Sengupta,9우 우 Solan district, $4 \sigma^{\top}$ Bergaon, Solan district, $1454 \mathrm{mt}$, 3055'14.2"N,7706'18.1"E, 18.iv.17, coll: J. Sengupta, 2 q $+30^{\pi} 0^{\pi}$ Bhagshung, Kangra district, $\quad 1755 \mathrm{mt}, \quad 32^{\circ} 14^{\prime} 23.1^{\prime \prime} \mathrm{N}$, 76¹9'41.3"E,5.vii.15, coll: J. Sengupta,2우 Palampur, Kangra district, $1220 \mathrm{mt}$, 326'39.09"N,76¹6'8.7"E,5.vii.15, coll: J. Sengupta, $20^{x} 0^{x}$ Bhagshung, Kangra district, 1755 mt, 32¹4'23.1"N, 76¹9'41.3"E,5.vii.15, coll: J. Sengupta,2 2 ㅇ Palampur, Kangra district, $1220 \mathrm{mt}, \quad 32^{\circ} 6^{\prime} 39.09^{\prime \prime} \mathrm{N}$, 76¹6'8.7"E,5.vii.15, coll: J. Sengupta, $80^{*} 0^{x}$ Chabutar Village, Hamirpur district, $774 \mathrm{mt}$, 31 $411^{\prime} 10.2^{\prime \prime} \mathrm{N}, 76^{\circ} 31^{\prime} 16.7^{\prime \prime E}$, 2.ii.16, coll: J. Sengupta, $50^{\pi} \sigma^{\pi}$ Karsai, Hamirpur district, 744 $\mathrm{mt}, 31^{\circ} 32^{\prime} 49.80^{\prime \prime} \mathrm{N}, 76^{\circ} 28^{\prime} 30.83 " \mathrm{E}, 3 . i \mathrm{i} .16$, coll: J. Sengupta, $50^{x} 0^{x}$ Galore, Hamirpur district, $720 \mathrm{mt}$,

31'38'56.54"N,76²5'10.53"E,3.ii.16,coll: J sengupta, $10^{*}$ Manman, Bilaspur district,847 $\mathrm{mt}, 31^{\circ} 17^{\prime} 55.87^{\prime \prime} \mathrm{N}, 76^{\circ} 45^{\prime} 55.77^{\prime \prime E}$, 2.ii.16,coll:
J sengupta, 1 i Kharkari, Bilaspur district, , 709mt, 31019'5.32"N, 76³2'53.81"E,2.ii.16,coll: J sengupta, $10^{*}$ Lahri, Bilaspur district, , 833mt, 31 ${ }^{\circ} 22^{\prime} 55.78^{\prime \prime} \mathrm{N}$, 76²7'46.79"E, 2.ii.16, coll: J Sengupta, 1 o Jahri, Bilaspur district, $791 \mathrm{mt}$, 31'27'28.32"N, 76²4'38.41"E, 2.ii.16,coll: J sengupta, $10^{*}$ Nalti, Bilaspur district, $722 \mathrm{mt}$, 31 $23^{\prime} 55.89^{\prime \prime} \mathrm{N}, \quad 76^{\circ} 45^{\prime} 22.52 " \mathrm{E} 2$.ii.16,coll: J sengupta, 1 i Guloti, Bilaspur district, $771 \mathrm{mt}$, 31'27'13.59"N, 76²8'44.88"E,2.ii.16,coll: J sengupta, $10^{\pi}$ Khauoo, Shimla district, $1739 \mathrm{mt}$, 3052'54.33"N, 77³8'17.49"E,, 20.iv.17, coll: J. Sengupta, $10^{\top}$ Manman, Bilaspur district, $847 \quad \mathrm{mt}, \quad 31^{\circ} 17^{\prime} 55.877^{\prime \prime} \mathrm{N}$, 76²5'55.77"E,8.ii.16,coll: J sengupta, $10^{\pi}$ Jahri, Bilaspur district, , $791 \mathrm{mt}$, 31'27'28.32"N,7640'38.41"E,2.ii.16,coll: J sengupta, $20^{\pi} \sigma^{\pi}$ Guloli, Bilaspur district, , 771 mt, 31 ${ }^{\circ} 27^{\prime} 13.59 " \mathrm{~N}, 7^{\circ} 28^{\prime} 44.88^{\prime \prime E}, 2 . i i .16$,coll: J sengupta, $30^{\pi} \sigma^{\pi}$ Majher, Bilaspur district, 1001 $\mathrm{mt}, 31^{\circ} 15^{\prime} 0.33^{\prime \prime N}, 76^{\circ} 49^{\prime} 54.74 " E, 4$.ii.16,coll: J sengupta, $40^{x} 0^{\pi}$ Kharkhari, Bilaspur district, , $709 \mathrm{mt}, \quad 31^{\circ} 19^{\prime} 5.32 " \mathrm{~N}, \quad$ 76³2'53.81"E, 8.ii.16,coll: J sengupta, $20^{x} 0^{x}$ Kharal, Kullu

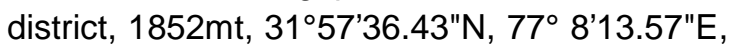
7.ii.16, coll: J. Sengupta, 4 우 ㅇ Hathol, Hamirpur district, 670mt, $31^{\circ} 41^{\prime} 35.55^{\prime \prime} \mathrm{N}$, 76²2'30.77"E, 4.ii.16, coll: J. Sengupta,4우우 Kajoti, Hamirpur district, $752 \mathrm{mt}$, 31'47'20.67"N, 76²8'47.87"E, 4.ii.16, coll: J. Sengupta,,2 9 Shishamati ,Kullu district, 1248 $\mathrm{mt}, 31^{\circ} 57^{\prime} 51.93^{\prime \prime} \mathrm{N}, 77^{\circ}$ 6'19.69"E, 7.ii.16, coll: J. Sengupta, $30^{\pi} 0^{\pi}$ Mohal, Kullu district, 1205 $\mathrm{mt}, 31^{\circ} 54^{\prime} 56.73^{\prime \prime} \mathrm{N}, 77^{\circ} 7{ }^{\prime} 1.58^{\prime \prime} \mathrm{E}, 7 . i i .16$, coll: J. Sengupta, 1 ㅇ Kullu, Kullu district, $1230 \mathrm{mt}$, 31 $577^{\prime 28.26 " N, ~} 77^{\circ}$ 6’34.05"E, 7.ii.16, coll: J. Sengupta, $30^{\star} 0^{x}$ Sarvari, Kullu district, $1202 \mathrm{mt}$, $31^{\circ} 57^{\prime} 37.15^{\prime \prime} \mathrm{N}, 77^{\circ} 6^{\prime} 47.23 " \mathrm{E}, 7 . i i .16$, coll: J. Sengupta, $10^{\pi}$ Dhalpur, Kullu district, $1212 \mathrm{mt}$,

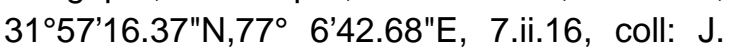
Sengupta, $20^{*} 0^{x}$ Gharakhar, Kullu district, 1436

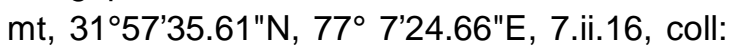
J. Sengupta,

Distribution: Himachal Pradesh, Arunachal Pradesh, Assam, Himachal Pradesh, Jammu \& Kashmir, Karnataka, Kerala, Manipur, Meghalaya, Mizoram, Nagaland, Orissa, Punjab, Sikkim, Tamil Nadu, Tripura, Uttarakhand, West Bengal. 
Elsewhere: Australasian Region (Australia), Oriental Region (Widely distributed), Palearctic Region (England).

\section{Genus Eupeodes OstenSacken, 1877}

Subgenus Macrosyrphus Matsumura, 1917

4. Eupeodes (Macrosyrphus) confrater (Wiedemann, 1830)

1830, Syrphus confrater Wiedemann, Auss. Zweifl. Theil. Schulz, Hamm. 12: 684

Type-locality: China

Material examined: 2 우 $90^{x} 0^{x}$ Bhagshung, Kangra district, $1755 \mathrm{mt}, 32^{\circ} 14^{\prime} 23.1{ }^{\prime \prime N}$, 76¹9'41.3"E,5.vii.15, coll: J. Sengupta

Distribution: India: Himachal Pradesh, Arunachal Pradesh, Assam, Bihar, Delhi, Gujarat,

Jammu \& Kashmir, Karnataka, Manipur, Meghalaya, Punjab, Sikkim, Tamil Nadu, Uttarakhand, West Bengal.

Elsewhere: Australasian Region (Australia, New Guinea), Oriental Region (Afghanistan, Nepal, Pakistan, Sri Lanka, Sumatra), Palaearctic Region (China).

Subgenus Metasyrphus Matsumura, 1917

5. Eupeodes (Metasyrphus) latifasciatus (Macquart, 1829)

1829. Syrphus latifasciatus Macquart, Sns.Dipt.N.Fr.4:94 (242).

Type locality: France.

Material examined: $10^{x} \mathrm{Khauoo,}$ Shimla district, $1739 \mathrm{mt}, 30^{\circ} 52^{\prime} 54.33 " \mathrm{~N}$

77³8'17.49"E, 20.iv.17, coll: J. Sengupta, $10^{\mathrm{X}}$ Bhagshung, Kangra district, 1755 mt, 32¹4'23.1"N, 76¹9'41.3"E,5.vii.15, coll: J. Sengupta

Distribution: India: Himachal Pradesh, Arunachal Pradesh, Jammu\& Kashmir, Nagaland, Uttarakhand.

Elsewhere: Australasian Region (Australatia), Oriental Region (Widely distributed), Palearctic Region (England, USA).
V. Genus Ischiodon Sack, 1913

6. Ischiodon scutellaris (Fabricius, 1805)

1805. Scaeva scutellaris Fabricius, Syst. Antliat.252.

Type-locality: Tranquebar, India.

Material examined: 1 if Dadahu Sirmour district, $\quad 651 \quad \mathrm{mt}, \quad 30^{\circ} 33^{\prime} 46.2456 " \mathrm{~N}$, 77²8'12.7086"E, 16.iv.17, coll: J. Sengupta,3우 우 Chachhi, Solan district, $890 \mathrm{mt}$, $31^{\circ} 2^{\prime} 40.15 " \mathrm{~N}, 76^{\circ} 52^{\prime} 37.85^{\prime \prime E}, 18 . i v .17$, coll: J. Sengupta, $30^{\pi} 0^{\pi}$ Kahnani, Solan district, 775 $\mathrm{mt}, \quad 31^{\circ} \quad 3^{\prime} 20.87^{\prime \prime N}, \quad 76^{\circ} 50^{\prime} 41.11$ "E,18.iv.17, coll: J. Sengupta, 2 우 ㅇ G Giana, Solan district, $881 \mathrm{mt}, \quad 31^{\circ} 13^{\prime} 31.82^{\prime \prime} \mathrm{N}, \quad 7^{\circ} 55^{\prime} 45.83 " \mathrm{E}$, 18.iv.17, coll: J. Sengupta, 1 + Bhanera, Solan district, $\quad 953 \quad \mathrm{mt}, \quad 31^{\circ} 38^{\prime} 0.65^{\prime \prime} \mathrm{N}$, 76³9'43.42"E,4.ii.16, coll: J. Sengupta, 4우 ㅇ Hathol, Hamirpur district, 670mt, 3141'35.55"N, 76²2'30.77"E, 4.ii.16, coll: J. Sengupta,4우우 Kajoti, Hamirpur district, 752mt, 3147'20.67"N, 76²8'47.87"E, 4.ii.16, coll: J. Sengupta, 2 우 ㅇ Renuka lake, Sirmour district, $672 \mathrm{mt}, 32^{\circ} 33^{\prime} 12.1^{\prime \prime} \mathrm{N}, 7^{\circ} 7^{\prime} 32.9^{\prime \prime} \mathrm{E}$, 15.iv.17, coll: J. Sengupta.

Distribution: Himachal Pradesh, Andhra Pradesh, Assam, Chandigarh, Delhi, Jammu \& Kashmir, Karnataka, Kerala, Madhya Pradesh, Manipur, Meghalaya, Orissa, Punjab, Rajasthan, Sikkim, Tamil Nadu, Tripura, Uttarakhand and West Bengal.

Elsewhere: Australasian Region (Australia, Pacific island), Oriental Region (Indonesia, Java), Palaearctic Region (Japan).

\section{Genus Meliscaeva Frey, 1946}

7. Meliscaeva cinctella (Zetterstedt, 1843)

1843, Scaeva cinctella Zetterstedt, Dipt. Scandin.1:742

Type-locality: Sweden. Ostergotland

Material examined: $20^{x} 0^{x}$ Majher, Bilaspur district, , $1001 \mathrm{mt}, 31^{\circ} 15^{\prime} 0.33^{\prime \prime} \mathrm{N}$, 7649'54.74"E, 4.ii.16,coll: J sengupta

Distribution: India: Himachal Pradesh, Meghalaya, Sikkim, West Bengal

Elsewhere: Oriental Region (Nepal), Palaearctic Region (China, Europe, USA). 
VII. Genus Scaeva Fabricius, 1805

8. Scaeva selenitica (Meigen, 1822)

1822. Syrphus selenticus Meigen, Syst. Baschr. Europ. Zweifl. Insekt.2:121.

Type-locality: Europe.

Material examined: 3 우 우 Chamor, Shimla district, $\quad 1714 \quad \mathrm{mt}, \quad 31^{\circ} 19^{\prime} 44.61^{\prime \prime} \mathrm{N}$, 77²2'48.18"E, 19.iv.17, coll: J. Sengupta,

Distribution: India: Himachal Pradesh, Arunachal Pradesh, Delhi, Meghalaya Sikkim, Uttarakhand, Uttar Pradesh.

Elsewhere: Palearctic Region (Alaska, China, Europe, France, Germany, Greenland, USA), Oriental Region (Japan).

9. Scaeva pyrastri (Linnaeus, 1758)**

1758.Musca pyrastri Linnaeus, Syst. Nat.10(1):594

Type-locality: Sweden.

Material examined: 2 우 Bergaon, Solan district, $1454 \mathrm{mt}, 30^{\circ} 55^{\prime} 14.2^{\prime \prime} \mathrm{N}, 7^{\circ} 06^{\prime} 18.1^{\prime \prime} \mathrm{E}$, 18.iv.17, coll: J. Sengupta, $10^{\top}$ Khauoo, Shimla district, $\quad 1739 \mathrm{mt}, \quad 30^{\circ} 52^{\prime} 54.33^{\prime \prime} \mathrm{N}$, 77³8'17.49"E,, 20.iv.17, coll: J. Sengupta

Distribution: India: Arunachal Pradesh, Delhi, Himachal Pradesh, Meghalaya and Uttar Pradesh.

Elsewhere: Palearctic Region (Alaska, Europe, France, Greenland, USA), Oriental Region (Japan).

Subgenus Sphaerophoria Wiedemann, 1830

10. Sphaerophoria(Sphaerophoria) indiana Bigot, 1884

1884. Sphaerophoria indiana Bigot, Annls. Soc. ent. Fr. (6) 4: 99

Type-locality: "Indes".

Material examined: $10^{\pi}$ Renuka Lake, Sirmour district, $650 \mathrm{mt}, \quad 32^{\circ} 33^{\prime} 12.11^{\prime \prime} \mathrm{N}$, 7607'32.9"E, 15.iv.17, coll: J.Sengupta, $20^{x} 0^{x}$ Bhagshung, Kangra district, $1755 \mathrm{mt}$, 32¹4'23.1"N, 76¹9'41.3"E,5.vii.15, coll: J. Sengupta, 2 우 우 Dadahu Sirmour district, 651 $\mathrm{mt}, 30^{\circ} 33^{\prime} 46.2456 " N .77^{\circ} 28^{\prime} 12.7086 " E 16 . i v .17$, coll: J. Sengupta, 1 i $20^{\pi} 0^{\pi}$ Bhagshung, Kangra district, $1755 \mathrm{mt}, \quad 32^{\circ} 14^{\prime} 23.1{ }^{\prime \prime} \mathrm{N}$, 76¹9'41.3"E,5.vii.15, coll: J. Sengupta,3우 우
Palampur, Kangra district, $1220 \mathrm{mt}$, 326'39.09"N, 76¹6'8.7"E,5.vii.15, coll: J. Sengupta,5우우 Palampur, Kangra district, $1220 \mathrm{mt}, 32^{\circ} 6^{\prime} 39.09$ "N, $^{\circ}$ 76¹6'8.7"E,5.vii.15, coll: J. Sengupta, $10^{\top}$ Manman, Bilaspur district, $847 \mathrm{mt}, \quad 31^{\circ} 17^{\prime} 55.87^{\prime \prime} \mathrm{N}, \quad 76^{\circ} 45^{\prime} 55.77^{\prime \prime} \mathrm{E}$, 8.ii.16,coll: J sengupta, $40^{\pi} \sigma^{\pi}$ Kharkhari, Bilaspur district, , $709 \mathrm{mt}, 31^{\circ} 19^{\prime} 5.32^{\prime \prime} \mathrm{N}$, 76³2'53.81"E, 8.ii.16,coll:J sengupta, $20^{x} 0^{x}$ Manman, Bilaspur district, , $833 \mathrm{mt}$, 31'22'55.78"N, 76²7'46.79"E, 8.ii.16,coll: J sengupta, $20^{x} 0^{x}$ Majher, Bilaspur district, , 1001 mt,31'15’0.33"N, 76²9'54.74"E, 4.ii.16,coll: J sengupta, $20^{x} 0^{x} 3$ 우 우 Bergaon, Solan district, $1452 \mathrm{mt}$, 30'54'16.1"N,775'48.2"E,18.iv.17, coll: J. Sengupta, 2 우 $30^{\pi} 0^{\pi}$ Bergaon, Solan district, 1454mt, 3055'14.2"N, 7706'18.1"E, 18.iv.17,

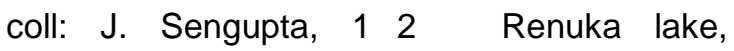
Sirmour district, $672 \mathrm{mt}, 32^{\circ} 33^{\prime} 12.1^{\prime \prime} \mathrm{N}$, 76³'32.9"E, 15.iv.17, coll: J. Sengupta,

Distribution: India: Himachal Pradesh, Arunachal Pradesh, Assam, Bihar, Delhi, Jammu \& Kashmir, Karnataka, Kerala, Maharashtra, Manipur, Meghalaya, Mizoram, Punjab, Sikkim, Tripura, Uttarakhand, Uttar Pradesh, West Bengal.

Elsewhere: Oriental Region (Bhutan, Myanmar, Nepal, Srilanka, Pakistan), Palearctic Region (Japan, Korea).

Subgenus Knutsonia Barkalov, 2012

$$
\begin{aligned}
& \text { 11. Sphaerophoria } \\
& \text { viridaenea Brunetti, } 1915
\end{aligned}
$$

1915, Sphaerophoria viridaenea, Rec. Indian Mus.11: 201-256

Type-locality: Himachal Pradesh: Shimla

Material examined: $20^{x} 0^{x} \quad 4$ 우 Hathol, Hamirpur district, 670mt, 31 ${ }^{\circ} 41^{\prime} 35.55^{\prime \prime} \mathrm{N}$, 76²2'30.77"E, 4.ii.16, coll: J. Sengupta, 4 우 우 Kajoti, Hamirpur district, 752mt, 31'47'20.67"N, 76²8'47.87"E, 4.ii.16, coll: J. Sengupta, 4 우우 Salouni, Hamirpur district, $791 \mathrm{mt}, 31^{\circ} 35^{\prime} 49.16 " \mathrm{~N}, 76^{\circ} 30^{\prime} 48.02 " \mathrm{E}, 4 . i i .16$, coll: J. Sengupta,

Distribution: India: Himachal Pradesh, Jammu \& Kashmir, Maharashtra, Mizoram, Punjab, Sikkim, Uttarakhand, West Bengal. 
Elsewhere: Oriental Region (Afghanistan, Pakistan, Nepal).

VIII. Genus Syrphus Fabricius, 1775

Subgenus Syrphus Fabricius, 1775

12. Syrphus(Syrphus) fulvifacies Brunetti, 1913

1913. Syrphus fulvifacies Brunetti; Rec.Ind. Mus,8:161.

Type-locality: Routung, NE Frontier, Assam, India.

Material examined: 4 우우 Palampur, Kangra district, $\quad 1220 \mathrm{mt}, \quad 32^{\circ} 6^{\prime} 39.09^{\prime \prime} \mathrm{N}$, 76¹6'8.7"E,5.vii.15, coll: J. Sengupta,4우우 Sahoo River side, Mandi district,930 mt, 32³3'12.1062"N, 727'32.9088"E, 24.iv.18, coll: J. Sengupta, 4 우우 $50^{\pi} 0^{\pi}$ Bergaon, Solan district, $1454 \mathrm{mt}, 30^{\circ} 55^{\prime} 14.2^{\prime \prime} \mathrm{N}, 77^{\circ} 06^{\prime} 18.1^{\prime \prime} \mathrm{E}$, 18.iv.17, coll: J. Sengupta,

Distribution: India: Assam, Arunachal Pradesh, Himachal Pradesh and Kashmir.

Elsewhere: China, Java, Nepal and Philippines.

IX. Genus Chrysotoxum Meigen,

13. Chrysotoxum convexam Brunetti, 1915

1915. Chrysotoxum convexum Brunetti, Rec. Indian Mus. 11: 249

Type-locality: Andarban, Garhwal (India: Uttarakhand).

Material examined: $60^{x} 0^{x} 5$ 우 Bergaon, Solan district, $1452 \mathrm{mt}, 30^{\circ} 54^{\prime} 16.1^{\prime \prime N}, 7^{\circ} 5^{\prime} 48.2^{\prime \prime} \mathrm{E}$, 18.iv.17, coll: J. Sengupta, $10^{\top} 1$ 우 Priunjal, Chamba district, $1712 \mathrm{mt}, 32^{\circ} 30^{\prime} 17.18^{\prime \prime} \mathrm{N}, 76^{\circ}$ 8'29.19"E, 18.iv.17, coll: J. Sengupta,

Distribution: India: Himachal Pradesh, Mizoram, Uttar Pradesh and Uttarakhand.

Elsewhere: Oriental Region (Pakistan), Palearctic Region (China).

14. violaceum Brunetti, 1923

Chrysotoxum

1923. Chrysotoxum violaceum Brunetti, Fauna. Br. India.3:302

Type-locality: Darjeeling, India.
Material examined: $20^{\pi} 0^{\pi} 2$ 우 우 Samot, Solan district, $1115 \mathrm{mt}, 31^{\circ} 5^{\prime} 43.20^{\prime \prime N}, 76^{\circ} 59^{\prime} 30.17 " \mathrm{E}$, 18.iv.17, coll: J. Sengupta,

Distribution: India: Himachal Pradesh, West Bengal.

Elsewhere: Nil.

Tribe Bacchini Bigot, 1883

X. Genus Baccha Fabricius, 1805

15. Baccha maculata Walker, 1852

1852. Baccha maculata Walker, Insecta Saundersiana..1: 223.

Type-locality: East Indies.

Material examined: $20^{\pi} 0^{\pi}$ Guloli, Bilaspur district, $771 \quad \mathrm{mt}, \quad 31^{\circ} 27^{\prime} 13.59^{\prime \prime} \mathrm{N}$, 76²8'44.88"E,2.ii.16,coll: J sengupta, 2 우 우 Renuka lake, Sirmour district, $672 \mathrm{mt}$, 32³3'12.1"N, 76²'32.9"E, 15.iv.17, coll: J. Sengupta, $10^{\top}$ Jahri, Bilaspur district, $791 \mathrm{mt}$, $31^{\circ} 27^{\prime} 28.32 " \mathrm{~N}$,

7640’38.41"E,2.ii.16,coll:JSengupta,

Distribution: India: Himachal Pradesh, Assam, Himachal Pradesh, Uttar Pradesh, West Bengal.

Elsewhere: Indo-Australian Region (Malaya, Philippines), Oriental Region (Borneo, Japan, Java, Kalimantan, Korea, Nepal, Sumatra, Taiwan), Palearctic Region (Russia).

XI. Genus Melanostoma Schiner, 1860

16. Melanostoma orientale (Wiedemann, 1824)

1824. Syrphus orientale Wiedemann. Analecta.Ent: 36.

Type-locality: "Ind. Or."

Material examined: 1ㅇ, Bhowen, Chamba

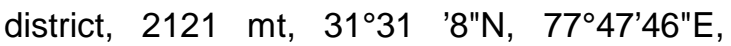
16.iv.17, coll: J.Sengupta, 4 우 우, $30^{x} \sigma^{x}$, Rampur power project, Kinnaur district, $970 \mathrm{mt}$, 31'23'38"N, 77³6'2"E, 14.iv.18, coll: J.Sengupta, 1ㅇ, Wangtoo, Kinnaur district, $1580 \mathrm{mt}, 31^{\circ} 33^{\prime} 48^{\prime \prime} \mathrm{N} \quad, \quad 77^{\circ} 59 \quad$ '30"E,

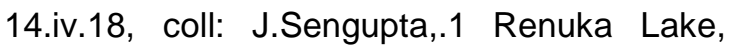
Sirmour district, 650mt, 32³3'12.1"N, 76³'32.9"E, 15.iv.17, coll: J.Sengupta. $30^{\pi} 0^{\pi} 3$ 우 우 Bergaon, Solan district, $1452 \mathrm{mt}$, 3054'16.1"N,775'48.2"E, 18.iv.17, coll: J. 
Sengupta $20^{\star}$ Guloli, Bilaspur district, , $771 \mathrm{mt}$, 3127'13.59"N,76²8'44.88"E,2.ii.16,coll: J sengupta, 4 우 우 Hathol, Hamirpur district,

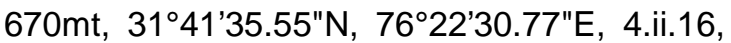
coll: J. Sengupta, 4 우 우 Kajoti, Hamirpur district, 752mt, 31'47'20.67"N, 76²8'47.87"E, 4.ii.16,

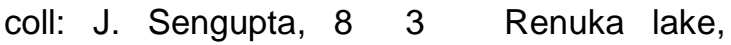
Sirmour district, $672 \mathrm{mt}, \quad 32^{\circ} 33^{\prime} 12.1 \mathrm{\prime N}$, 76³'32.9"E,15.iv.17, coll: J. Sengupta, $50^{\pi} 0^{\top} 9$ 우 우 Nurchok, Mandi district, $784 \mathrm{mt}$, $31^{\circ} 36^{\prime} 30.6792 " \mathrm{~N}, 76^{\circ} 54^{\prime} 55.1808 " \mathrm{E}, 15 . i v .18$, coll: J. Sengupta,4우우 Palampur, Kangra district, $\quad 1220 \quad \mathrm{mt}, \quad 32^{\circ} 6^{\prime} 39.09^{\prime \prime} \mathrm{N}$, 76¹6'8.7"E,5.vii.15, coll: J. Sengupta,1우 Chabutar Village, Hamirpur district, $774 \mathrm{mt}$, 3141'10.2"N, 76³1'16.7"E, 2.ii.16, coll: J. Sengupta,4 아 Hathol, Hamirpur district, $670 \mathrm{mt}$, 314'3․55"N, 76²2'30.77"E, 3.ii.16, coll: J. Sengupta, $10^{x}$ Bahli, Shimla district, $1634 \mathrm{mt}$, 31' 9'17.81"N, 7743'47.08"E,, 20.iv.17, coll: J. Sengupta, $10^{x}$ Jahri, Bilaspur district, , $791 \mathrm{mt}$, 31'27'28.32"N, 76²0'38.41"E,2.ii.16,coll: J sengupta,

Distribution: India: Assam, Arunachal Pradesh, Himachal Pradesh, J \& K, Karnataka, Meghalaya, Sikkim, T.N, Tripura, Uttar Pradesh, and West Bengal.

Elsewhere: Oriental Region (Bhutan, Nepal, Pakistan, Sri Lanka)

Tribe Paragini Glumac, 1961

XII. Genus Paragus Latreille, 1804

Subgenus Paragus Latreille, 1804

$$
\begin{aligned}
& \text { 17. Paragus (Paragus) serratus } \\
& \text { (Fabricius, 1805) }
\end{aligned}
$$

1805. Mulio serratus Fabricius, Syst. Antliat.3:186

Type locality: Tamil Nadu: Tharangambadi, India.

Material examined: $20^{\pi} 0^{\pi}$ Amroh, Hamirpur district, $764 \mathrm{mt}, 31^{\circ} 43^{\prime} 8.71^{\prime \prime N}, 76^{\circ} 28^{\prime} 45.82^{\prime \prime} \mathrm{E}$, 4.ii.16, coll: J. Sengupta,5우 ㅇ Suhin, Una district, $701 \mathrm{mt}, 31^{\circ} 45^{\prime} 18.46^{\prime \prime} \mathrm{N}, 76^{\circ} 6^{\prime} 47.299^{\prime \prime} \mathrm{E}$, 12.ii.16, coll: J. Sengupta, $20^{\pi} 0^{x}$ Bhanera, Hamirpur district, $953 \mathrm{mt}, 31^{\circ} 38^{\prime} 0.65^{\prime \prime} \mathrm{N}$, 76³9'43.42"E, 4.ii.16, coll: J. Sengupta,

Distribution: India: Himachal Pradesh, Assam, Bihar, Delhi, Jammu \& Kashmir, Karnataka, Kerala, Madhya Pradesh,
Maharashtra, Manipur, Meghalaya, Orissa, Punjab, Tamil Nadu, Tripura, Uttar Pradesh, West Bengal)

Distribution: Elsewhere: Oriental Region (Africa, Borneo, Java, Nepal, Pakistan, Papuya, Sri Lanka)

Subfamily ERISTALINAE

Tribe Rhingiini

XIII. Genus Cheilosia Meigen, 1822

18. Cheilosia nigroaenea Brunetti, 1915

1915. Chilosia nigroaenea Brunetti, Rec.Indian Mus, 11:204

Type locality: India, Matiana \& Simla.

Material examined: 6 우 $40^{x} 0^{x}$ Dariaghat, Solan district,931 mt, $31^{\circ} 15^{\prime} 17.50 " \mathrm{~N}$, 7655'42.90"E, 18.iv.17, coll:J.Sengupta, 3 우 우 $20^{\pi} 0^{x}$ Sultej river side, Solan district, 1018 $\mathrm{mt}$, 3120'27.49"N, 7653'26.63"E, 18.iv.17, coll: J. Sengupta

Distribution: India: Himachal Pradesh and Uttar Pradesh.

Elsewhere: Oriental Region (Nepal)

Tribe Volucellini Newman, 1834

XIV. Genus Volucella Geoffroy, 1762

19. Volucella ruficauda Brunetti, 1907

1907. Volucella ruficauda Brunetti, Rec. Indian Mus.1: pl. xi, fig.13; 1908

Type-locality: Sikkim, India.

Material examined: 2 우우, Choling garden, Kinnaur district, $1780 \mathrm{mt}, 31^{\circ} 30^{\prime} 60^{\prime \prime} \mathrm{N}, 78^{\circ} 9$ '9"E,13.iv.18, coll: J.Sengupta,1 9 , Tapri garden side, Kinnaur district, $1600 \mathrm{mt}, 31^{\circ} 32^{\prime} 5^{\prime \prime N}, 78^{\circ} 1$ '8"E, 13.iv.18, coll: J.Sengupta, 2 우 오 Shong Thong, Kinnaur district, $1910 \mathrm{mt}, 31^{\circ} 31^{\prime} 8 " \mathrm{~N}$, 78 16'13"E, 13.iv.18, coll: J.Sengupta, $40^{x} 0^{x}$ Chhob, Solan district, $1311 \mathrm{mt}, 30^{\circ} 55^{\prime} 38.28^{\prime \prime} \mathrm{N}$, 77¹1'52.38"E, 18.iv.17, coll: J. Sengupta.

Distribution: India: Himachal Pradesh, Sikkim.

Elsewhere: Nil.

XV. Genus Sphiximorpha Rondani, 1850 
20. Sphiximorpha triangulifera (Brunetti, 1913)

1913. Ceria triangulifera Brunetti, Rec. Indian Mus.9: 273

Type locality: Darjeeling District, India.

Material examined: 2 우우, Choling garden, Kinnaur district, $1780 \mathrm{mt}, 31^{\circ} 30^{\prime} 60^{\prime \prime} \mathrm{N}, 78^{\circ} 9$ '9"E,13.iv.18, coll: J.Sengupta, 1 \%, Tapri garden side, Kinnaur district, $1600 \mathrm{mt}, 31^{\circ} 32^{\prime} 5^{\prime \prime N}, 78^{\circ} 1$ '8"E, 13.iv.18, coll: J.Sengupta, 2 우, Shong Thong, Kinnaur district, $1910 \mathrm{mt}, 31^{\circ} 31^{\prime} 8 \mathrm{~B} \mathrm{~N}$, 78'16'13"E, 13.iv.18, coll: J.Sengupta, 40 Chhob, Solan district, $1311 \mathrm{mt}, 30^{\circ} 55^{\prime} 38.28^{\prime \prime} \mathrm{N}$, 77¹1'52.38"E, 18.iv.17, coll: J. Sengupta,

Distribution: India: Himachal Pradesh, West Bengal

Elsewhere: NIL.

Tribe Eristalini Newman, 1834

XVI. Genus Eristalinus Rondani, 1845

21. Eristalinus (Eristalinus) arvorum (Fabricius, 1787)

1787.Syrphus arvorum Fabricius, Mantissa insectorum. 2(2): 335

Type-locality: India. Tamil Nadu: Tharangambadi.

Material examined: 1 우 Bhanera, Solan district, $953 \mathrm{mt}, 31^{\circ} 38^{\prime} 0.65^{\prime \prime} \mathrm{N}, 76^{\circ} 39^{\prime} 43.42^{\prime \prime} \mathrm{E}$, 4.ii.16, coll: J. Sengupta, 2 우 ㅇ Bergaon, Solan district, $1454 \mathrm{mt}, 30^{\circ} 55^{\prime} 14.2^{\prime \prime} \mathrm{N}, 7^{\circ} 06^{\prime} 18.1 " \mathrm{E}$, 18.iv.17, coll: J. Sengupta,4우우 Palampur, Kangra district, $1220 \mathrm{mt}, 32^{\circ} 6^{\prime} 39.09^{\prime \prime} \mathrm{N}$, 76¹6'8.7"E, 5.vii.15, coll: J. Sengupta,3우우 Salouni, Hamirpur district, $791 \mathrm{mt}$, 31'35'49.16"N, 76³0'48.02"E, 3.ii.16, coll: J. Sengupta,4우우 Hathol, Hamirpur district, 670mt, 31ํ1'35.55"N, 76²2'30.77"E, 4.ii.16, coll: J. Sengupta, 4 우 Kajoti, Hamirpur district, 752mt, 3104'20.67"N, 76²8'47.87"E, 4.ii.16, coll: J. Sengupta.

Distribution: India: Himachal Pradesh, Tamil Nadu, West Bengal.

Elsewhere: Afro tropical region (Seychelles), Australasian region(Australia), Palearctic Region (China, Japan)

22. Eristalinus (Eristalinus)
megacephalus (Rossi,1794)

1794. Syrphusmegacephalus Rossi, Mantissa. insect. 2:3

Type-locality: Italy. Toscana.

Material examined: $10^{\pi}$ Bergaon, Solan district, $1452 \mathrm{mt}, 30^{\circ} 54^{\prime} 16.1^{\prime \prime N}, 7^{\circ} 5^{\prime} 48.2^{\prime \prime} \mathrm{E}$, 18.iv.17, coll: J. Sengupta, 1 i Chabutar village, Hamirpur district, $774 \mathrm{mt}$, 31'41'10.2"N, 76³1'16.7"E, 02.ii.16, coll: J. Sengupta.

Distribution: India: Himachal Pradesh, Andhra Pradesh, Bihar, Gujarat, Jammu \& Kashmir, Karnataka, Kerala, Maharashtra, Meghalaya, Orissa, Rajasthan, Sikkim, Tamil Nadu, Tripura, Uttarakhand, West Bengal

Elsewhere: Indo Australian region (Singapore), Oriental Region (Afghanistan, BuruNepal, Pakistan, Sri Lanka), Palearctic Region (China, Japan).

23. Eristalinus (Eristalinus) polychromata $($ Brunetti, 1923) **

1923. Eristalis polychromata Brunetti, Fauna of British India.3: 180

Type locality: India: Kolkata.

Material examined: $10^{x}$ Chabutar Village, Hamirpur district, $774 \mathrm{mt}$, 31²41'10.2"N,76³1'16.7"E, 2.ii.16, coll: J. Sengupta, 1 ㅇ Ghiana, Solan district, $881 \mathrm{mt}$, 31'13'31.82"N,7655'45.83"E,18.iv.17, coll: J. Sengupta, 4 우 ㅇ Hathol, Hamirpur district, 670mt, 31'41'35.55"N, 76²2'30.77"E, 4.ii.16, coll: J. Sengupta, 4 우 ㅇ Kajoti, Hamirpur district, 752mt, 31²4'20.67"N, 76²8'47.87"E, 4.ii.16, coll: J. Sengupta,

Distribution: India: Himachal Pradesh, West Bengal

Elsewhere: Nil.

24. Eristalinus (Eristalinus) tabanoides (Jaennicke, 1867)

1867. Eristalis tabanoides Jaennicke, Neu. exot. Dipt. Abh. Ges.6: 402

Type-locality: Eritrea.

Material examined: $10 \circ$ ㅇ, Parla, Una district, $647 \mathrm{mt}, \quad 31^{\circ} 27^{\prime} 15.00^{\prime \prime} \mathrm{N}, \quad 76^{\circ} 24^{\prime} 10.98^{\prime \prime} \mathrm{E}$, 04.ii.16, coll: J.Sengupta, $30^{\pi} \sigma^{\pi}$ Nurchok, Mandi district, 784mt, $31^{\circ} 36^{\prime} 30.6^{\prime \prime} \mathrm{N}$, 7654'55.1"E, 26.iv.18, coll: J. Sengupta, 
$30^{\pi} \sigma^{x} 1$ ㅇ Bergaon, Solan district, $1452 \mathrm{mt}$, $30^{\circ} 54^{\prime} 16.1 " \mathrm{~N}, 77^{\circ} 5^{\prime} 48.2^{\prime \prime} \mathrm{E}, 18 . i v .17$, coll: J. Sengupta.

Distribution: India: Himachal Pradesh, Delhi.

Elsewhere: Afro tropical region (Eritrea, Djibouti), Palearctic region (Egypt, Tunisia).

\section{Eristalinus (Eristalinus) quinquestriatus (Fabricius, 1794)}

1794. Syrphus quinquestriatus Fabricius, Ent. syst.4: 289

Type-locality: Tamil Nadu: Tharangambadi.

Material examined: 10 우, Parla, Una district, $647 \mathrm{mt}, \quad 31^{\circ} 27^{\prime} 15.00^{\prime \prime} \mathrm{N}, \quad 7^{\circ} 24^{\prime} 10.98^{\prime \prime} \mathrm{E}$, 04.ii.16, coll: J.Sengupta, $3 \sigma^{x} \sigma^{x}$ Nurchok,Mandi district, 784mt, 31 $36^{\prime} 30.6^{\prime \prime} \mathrm{N}, \quad 76^{\circ} 54^{\prime} 55.1^{\prime \prime} \mathrm{E}$, 26.iv.18, coll: J. Sengupta,30 1 우 Bergaon, Solan district, $1452 \mathrm{mt}, \quad 30^{\circ} 54^{\prime} 16.1{ }^{\prime \prime} \mathrm{N}$, 775'48.2"E, 18.iv.17, coll: J. Sengupta.

Distribution: India: Himachal Pradesh, Arunachal Pradesh, Assam, Bihar, Karnataka, Kerala, Madhya Pradesh, Nagaland, Orissa, Tripura, Uttar Pradesh, Uttarakhand and West Bengal.

Elsewhere: Oriental Region (Buru, China, Indonesia, Japan)

Subgenus Eristalodes Mik, 1897

26. Eristalinus (Eristalodes) taeniops (Wiedemann, 1818)

1818. Eristalis taeniops Wiedemann, Zool. Mag.1(2): 42

Type-Iocality: South Africa. Cape.

Material examined: 2 우 Dadahu Sirmour district, $651 \quad \mathrm{mt}, \quad 30^{\circ} 33^{\prime} 46.2456 " \mathrm{~N}$, 77²8'12.7086"E, 16.iv.17, coll: J. Sengupta,2우 웅 Ghiana, Solan district, $881 \mathrm{mt}$, $31^{\circ} 13^{\prime} 31.82^{\prime \prime} \mathrm{N}, 76^{\circ} 55^{\prime} 45.83^{\prime \prime E}$, 18.iv.17, coll: J. Sengupta,,6우 우 Swarghat, Solan district, 816mt, 3113'57.29"N, 76²4'27.25"E, 18.iv.17, coll: J. Sengupta,: 8 우 ㅇ Renuka lake, Sirmour district, $672 \mathrm{mt}, 32^{\circ} 33^{\prime} 12.1^{\prime \prime} \mathrm{N}$, 767’32.9"E, 15.iv.17, coll: J. Sengupta.

Distribution: India: Himachal Pradesh, Arunachal Pradesh, Chandigarh, Jammu \& Kashmir Karnataka, Manipur, Meghalaya, Mizoram, Sikkim, Tamil Nadu, Uttarakhand, Uttar Pradesh, West Bengal.
Elsewhere: Afro Tropical Region (Africa), Oriental Region (Afghanistan, Nepal, Pakistan, Sri Lanka, Taiwan), Indo-Australian Region (Java, Moluccas), Palearctic Region (Europe, USA)

27. Eristalinus (Eristalodes) paria (Bigot, 1880)

1880. Eristalomyia paria Bigot, Ann. Soc. Ent. Fr. ser. 5(10): 218

Type-locality: Sri Lanka.

Material examined: 1 if Chabutar village, Hamirpur district, $774 \mathrm{mt}, 31^{\circ} 41^{\prime} 10.2^{\prime \prime} \mathrm{N}$, 76³1'16.7"E, $\quad$ 01.ii.16,coll: J.Sengupta, $10^{x}$ Palampur, Kangra district, 1220 mt, 326'39.09"N, 76¹6'8.7"E,5.vii.15, coll: J. Sengupta, 1 ㅇ Bhagshung, Kangra district, 1755 mt, 32॰14'23.1"N, 76¹9'41.3"E,5.vii.15, coll: J. Sengupta, 2 우 우 Chabutar village, Hamirpur district, $774 \mathrm{mt}, 31^{\circ} 41^{\prime} 10.2^{\prime \prime} \mathrm{N}$, 76³1'16.7"E, 02.ii.16,coll: J. Sengupta, $20^{x} 0^{x}$ Nurchok, Mandi district, $784 \mathrm{mt}$, 31³6'30.6792"N, 7654'55.1808"E, 25.iv.18, coll: J. Sengupta, $30^{\pi} 0^{\pi}$ Bhagpur, Mandi district, $\quad 855 \mathrm{mt}, 31^{\circ} 17^{\prime} 37.6866 \mathrm{~N} \mathrm{~N}$, 7652'14.3574"E, 23.iv.18, coll: J. Sengupta, $30^{x} 0^{x} 3$ 우 우 Borapani, Solan district, $1524 \mathrm{mt}, 30^{\circ} 55^{\prime 2} 23.8^{\prime \prime} \mathrm{N}, 7^{\circ} 06^{\prime} 19.6 " \mathrm{E}, 18 . i v .18$, coll: J. Sengupta, $2 \sigma^{\pi} \sigma^{\pi}$ Bergaon, Solan district, $1454 \mathrm{mt}, 30^{\circ} 55^{\prime} 14.2^{\prime \prime} \mathrm{N}, 77^{\circ} 06^{\prime} 18.1$ "E18.iv.17, coll: J. Sengupta,

Distribution: India: Himachal Pradesh, Arunachal Pradesh, Chandigarh, Jammu \& Kashmir, Karnataka, Manipur, Meghalaya, Mizoram, Sikkim, Tamil Nadu, Uttarakhand, Uttar Pradesh, West Bengal.

Elsewhere: Oriental Region (Sri Lanka, Taiwan), Indo-Australian Region (Java, Moluccas), Palearctic Region (China)

XVII. Genus Eristalis Latreille, 1804

Subgenus Eoseristalis Kanervo, 1938

28. Eristalis(Eoseristalis) albibasis Bigot, 1880

1880. Eristalis albibasis Bigot, Annls Soc. Ent Fr. (5) 10: 215.

Type-Iocality: "Indostan" 
Material examined: $20^{x} 0^{x}$ Khauoo, Shimla district, $1739 \mathrm{mt}, \quad 30^{\circ} 52^{\prime} 54.33 " \mathrm{~N}$, 77³8'17.49"E,, 20.iv.17, coll: J. Sengupta.

Distribution: India: Himachal Pradesh.

Elsewhere: Palearctic region (China).

\section{Eristalis (Eoseristalis) arbustorum (Linnaeus, 1758)}

1758. Musca arbustorumLinnaeus, Syst. Nat. Ed. 10: 591.

Type-locality: Sweden.

Material examined: $10^{\pi}$ Bhagshung, Kangra district, $\quad 1755 \mathrm{mt}, \quad 32^{\circ} 14^{\prime} 23.1{ }^{\prime \prime} \mathrm{N}$, 76¹9'41.3"E,5.vii.15, coll: J. Sengupta, 2 우 Palampur, Kangra district, $1220 \mathrm{mt}$, 326'39.09"N, 76¹6'8.7"E,5.vii.15, coll: J. Sengupta, $10^{*}$ Bhagshung, Kangra district, $1755 \mathrm{mt}, 32^{\circ} 14^{\prime} 23.1 " \mathrm{~N}, 76^{\circ} 19^{\prime} 41.3 " \mathrm{E}, 5 . v i i .15$, coll: J. Sengupta,

Distribution: India: Himachal Pradesh, Arunachal Pradesh, Jammu \& Kashmir, Meghalaya, Sikkim, West Bengal.

Elsewhere: Afrotropical Region (Africa), Palaearctic Region (Canada, China, Europe, Japan, Siberia, USA), Oriental Region (Afghanistan, Pakistan).

\section{Eristalis(Eoseristalis) cerealis} Fabricius, 1805

1805. Eoseristalis cerealis Fabricius, Syst. Antliat. 14: 232.

Type-locality: China.

Material examined: $10^{\pi}$ Renuka Lake, Sirmour district, 650mt, 32०33'12.1"N, 7607'32.9"E, 15.iv.17, coll: J.Sengupta, $10^{\pi}$ Renuka Lake, Sirmour district, $672 \mathrm{mt}$, 32०33'12.1"N, 767'32.9"E, 15.iv.17, coll: J.Sengupta, $10^{*}$ Chabutar Village, Hamirpur district, 649mt, $31^{\circ} 41^{\prime} 10.2^{\prime \prime} \mathrm{N}, \quad 76^{\circ} 41^{\prime} 14.8^{\prime \prime} \mathrm{E}$, 02.ii.16, coll: J.Sengupta, 4 ㅇ $+70^{\pi} 0^{\pi} \mathrm{Jamli}$, Bilaspur district, , 685mt, 30³3'46.2456"N ,77²8'12.7086"E, 15.iv.17, coll: J. Sengupta, $50^{x} 0^{x}$ Chabutar Village, Hamirpur district, 684 $\mathrm{mt}, 31^{\circ} 41^{\prime} 10.2^{\prime \prime} \mathrm{N}, 76^{\circ} 41^{\prime} 14.8^{\prime \prime E}, 2 . i i .16$, coll: J. Sengupta,4우 ㅇ Chabutar Village, Hamirpur district, $\quad 774 \quad \mathrm{mt}, \quad 31^{\circ} 41^{\prime} 10.2^{\prime \prime} \mathrm{N}$, 76³1'16.7"E,1.ii.16, coll: J. Sengupta, 2 우 우
Dadahu Sirmour district, $651 \mathrm{mt}$, 30³3'46.2456"N.77²8'12.7086"E16.iv.17, coll: J. Sengupta, $30^{\pi} \sigma^{\pi}$ Bahli, Shimla district, $1634 \mathrm{mt}, 31^{\circ} 9^{\prime} 17.81 " \mathrm{~N}, 7^{\circ} 43^{\prime} 47.08^{\prime \prime} \mathrm{E}$, 20.iv.17, coll: J. Sengupta, $10^{\top}$ Manman, Bilaspur district, , $847 \mathrm{mt}, 31^{\circ} 17^{\prime} 55.87^{\prime \prime} \mathrm{N}$, 76 $45^{\prime}$ '55.77"E,8.ii.16,coll: J sengupta, $60^{\pi} 0^{\pi}$ Nurchok, Mandi district, $784 \mathrm{mt}$, 31'36’30.6792"N, 7654'55.1808"E, 15.iv.18, coll: J. Sengupta, 3 \% Mandi, Mandi district, $1023 \mathrm{mt}, 31^{\circ} 43^{\prime} 51.5^{\prime \prime} \mathrm{N}, 76^{\circ} 56^{\prime} 30.4^{\prime \prime E}, 24 . i v .18$, coll: J. Sengupta, 6 우 ㅇ Bergaon, Solan district, 1454mt, 3055'14.2"N,7706'18.1"E, 18.iv.17, coll: J. Sengupta, 2 ㅇ ㅇ Bergaon, Solan district, $1452 \mathrm{mt}, 30^{\circ} 54^{\prime} 16.1^{\prime \prime} \mathrm{N}, 77^{\circ} 5^{\prime} 48.2^{\prime \prime} \mathrm{E}, 18 . i v .17$, coll: J. Sengupta,1 1 Ghiana, Solan district, $881 \quad \mathrm{mt}, \quad 31^{\circ} 13^{\prime} 31.82^{\prime \prime} \mathrm{N}$, 7655'45.83"E,18.iv.17, coll: J. Sengupta,,6우 우 Swarghat, Solan district, $816 \mathrm{mt}$, 31'13'57.29"N, 7643'27.25"E, 18.iv.17, coll: J. Sengupta,: 8 우 Renuka lake, Sirmour district, $672 \mathrm{mt}, 32^{\circ} 33^{\prime} 12.1^{\prime \prime} \mathrm{N}, 76^{\circ} 7^{\prime} 32.9^{\prime \prime E}, 15 . i v .17$, coll: J. Sengupta, $10^{\pi}$ Dhalpur, Kullu district, $1212 \mathrm{mt}, 31^{\circ} 57^{\prime} 16.37^{\prime \prime N}, 77^{\circ}$ 6'42.68"E,7.ii.16,, coll: J. Sengupta, $20^{x} 0^{x}$ Gharakhar, Kullu district, $1436 \mathrm{mt}, 31^{\circ} 57^{\prime} 35.61^{\prime \prime N}, 77^{\circ} 7$ '24.66"E, 7.ii.16, coll: J. Sengupta, $40^{\pi} 0^{\pi}$ Kharkhari, Bilaspur district, $709 \mathrm{mt}, 31^{\circ} 19^{\prime} 5.32 \mathrm{~N} \mathrm{~N}$, 76³2'53.81"E, 8.ii.16,coll: J sengupta, $20^{x} \sigma^{x}$ Majher, Bilaspur district, $1001 \mathrm{mt}$, 31'15'0.33"N, 76²99'54.74"E, 4.ii.16,coll: J sengupta,2 2 o Shishamati ,Kullu district, 1248 $\mathrm{mt}$, 31 ${ }^{\circ} 57^{\prime} 51.93^{\prime \prime} \mathrm{N}, 77^{\circ}$ 6'19.69"E, 4.ii.16, coll: J. Sengupta,, 1 \% Mohal ,Kullu district, $1205 \mathrm{mt}$,

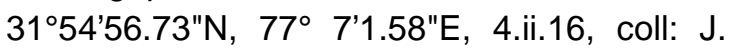
Sengupta, 1 ㅇ Kullu,Kullu district, $1230 \mathrm{mt}$, 31'57'28.26"N, 776'34.05"E, 5.vii.15, coll: J. Sengupta, $3 \sigma^{\pi} \sigma^{\pi}$ Sarvari,Kullu district, $1202 \mathrm{mt}$, 31 $57^{\prime} 37.15^{\prime \prime} \mathrm{N}, \quad 77^{\circ} 6^{\prime} 47.23 " \mathrm{E}, 7 . i i .16$, coll: J. Sengupta, 1 우 Chabutar Village, Hamirpur district, $\quad 774 \quad \mathrm{mt}, \quad 31^{\circ} 41^{\prime} 10.2^{\prime \prime} \mathrm{N}$, 76³1'16.7"E,2.ii.16, coll: J. Sengupta,

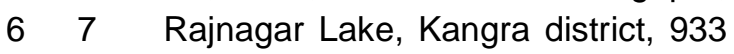
mt, 32³7'26.5548"N, 76³'49.8522"E, 5.vii.15, coll: J. Sengupta.

Distribution: India: Himachal Pradesh, Arunachal Pradesh, Jammu \& Kashmir, Manipur, Meghalaya, Mizoram, Nagaland, ,Sikkim, Tamil Nadu, Uttarakhand, West Bengal.

Elsewhere: Oriental region (Myanmar,Nepal), Palearctic region (China, Japan,Russia) 


\section{Eristalis(Eoseristalis) himalayensis Brunetti, 1908}

1908. Eristalis himalayensis Brunetti, Rec. Indian Mus. 2: 70

Type-locality: "Indostan"

Material examined: $20^{\pi} 0^{\pi}$ Gharakhar, Kullu district, $1436 \mathrm{mt}, 31^{\circ} 57^{\prime} 35.611^{\prime \prime} \mathrm{N}, 77^{\circ} 7^{\prime} 24.66 " \mathrm{E}$, 7ii.16, coll: J. Sengupta,2 $0^{x} 0^{x} 2$ 우 ㅇ Khauoo, Shmla district, $1739 \mathrm{mt}, \quad 30^{\circ} 52^{\prime} 54.33^{\prime \prime} \mathrm{N}$, 77³8'17.49"E, 19.iv.17, coll: J. Sengupta, 30 $0^{\pi}$ Nurchok, Mandi district, $785 \mathrm{mt}$, 31'36’30.6"N, 7654'55.1"E, 26.iv.18, coll: J. Sengupta.

Distribution: India: Himachal Pradesh, Arunachal Pradesh, Jammu \& Kashmir, Sikkim, Uttar Pradesh, Uttarakhand, West Bengal

Elsewhere: Oriental Region (China, Java, Myanmar, Nepal Sri Lanka). Indo-Australian Region (Malaya, Philippines, Sumatra, Sumbawa.)

Subgenus Eristalis Latreille, 1804

32. Eristalis (Eristalis) tenax (Linnaeus, 1758)

1758, Musca tenax Linnaeus, syst. Nat. 10 (1): 591.

\section{Type-locality: Europe}

Material examined: 1 우 Sarahan village panchayet area, Kinnaur district, $1710 \mathrm{mt}$, $31^{\circ} 31^{\prime} 39^{\prime \prime N}, 77^{\circ} 47$ '16"E, 13.iv.18, coll: J. $30^{x} 0^{x}, 1$ + Renuka Lake, Sirmour district, $650 \mathrm{mt}$, 32॰33'12.1"N, 767'32.9"E, 15.iv.17, coll: J. Sengupta, 3 우 Khajjiar, Chamba district, 1809mt, 32³3'20.8"N, 76³'56.09"E, 04.vii.15, coll: J. Sengupta, $10^{\lambda} 2$ 우우 Chachhi, Solan district, $\quad 890 \quad \mathrm{mt}, \quad 31^{\circ}$ 2'40.15"N,7652'37.85"E,18.iv.17, coll: J. Sengupta, $30^{x} 0^{x} 2$ 우 + Kahnani, Solan district, $775 \mathrm{mt}, 31^{\circ} 3^{\prime} 20.87^{\prime \prime} \mathrm{N}, 76^{\circ} 50^{\prime} 41.11$ "E,18.iv.17, coll: J. Sengupta, $10^{*}$ Ghiana, Solan district, $881 \mathrm{mt}, \quad 31^{\circ} 13^{\prime} 31.82^{\prime \prime} \mathrm{N}, \quad 76^{\circ} 55^{\prime} 45.83^{\prime \prime} \mathrm{E}$, 18.iv.17, coll: J. Sengupta, $50^{\pi} 0^{\pi} 7$ 우 ㅇD.P.F. SAGAN, Solan district, $1005 \mathrm{mt}, 31^{\circ} 20^{\prime} 5.09^{\prime \prime N}$, 7652'21.71"E, 18.iv.17, coll: J. Sengupta, $70^{\pi} 0^{\pi} 4$ 우 우 Bergaon, Solan district, $1452 \mathrm{mt}, 30^{\circ} 54^{\prime} 16.1 " \mathrm{~N}, 7^{\circ} 5^{\prime} 48.2 " \mathrm{E}, 18 . i v .17$, coll: J. Sengupta, 3 우 ㅇ Bergaon, Solan district, $1452 \mathrm{mt}, 30^{\circ} 54^{\prime} 16.1^{\prime \prime} \mathrm{N}, 77^{\circ} 5^{\prime} 48.2^{\prime \prime E}, 18 . i v .17$, coll: J. Sengupta, $20^{\pi} 0^{\pi} 1$ ㅇ Chachhi, Solan district, $890 \quad \mathrm{mt}, \quad 31^{\circ} \quad 2^{\prime} 40.15 " \mathrm{~N}$, 7652'37.85"E,18.iv.17, coll: J. Sengupta, $20^{x} 0^{x} 3$ 우 우 Kahnani, Solan district, $775 \mathrm{mt}, \quad 31^{\circ} \quad 3^{\prime} 20.87^{\prime \prime} \mathrm{N}, \quad 76^{\circ} 50^{\prime} 41.11^{\prime \prime} \mathrm{E}$, 18.iv.17, coll: J. Sengupta, 1 + Ghiana, Solan district, $\quad 881 \quad \mathrm{mt}, \quad 31^{\circ} 13^{\prime} 31.82^{\prime \prime} \mathrm{N}$, 7655'45.83"E,18.iv.17, coll: J. Sengupta, 6 우 우 Bergaon, Solan district, $1452 \mathrm{mt}$, $30^{\circ} 54^{\prime} 16.1 " \mathrm{~N}, 7^{\circ} 5^{\prime} 48.2^{\prime \prime} \mathrm{E}$, 18.iv.17, coll: J. Sengupta, $70^{\pi} 0^{\pi} 7$ 우 우 Bergaon, Solan district, $1454 \mathrm{mt}, 30^{\circ} 54^{\prime} 16.1^{\prime \prime} \mathrm{N}, 7^{\circ} 5^{\prime} 48.2^{\prime \prime} \mathrm{E}, 18 . i v .17$, coll: J. Sengupta, $10^{\top} 3$ 우 울 Chamor, Solan district, $\quad 1714 \mathrm{mt}, \quad 31^{\circ} 19^{\prime} 44.61^{\prime \prime} \mathrm{N}$, 77²2'48.18"E, 19.iv.17, coll: J. Sengupta, $15 \sigma^{\pi} \sigma^{\top} 22$ 우 ㅇ Palampur, Kangra district, $\quad 1220 \mathrm{mt}, \quad 32^{\circ} 6^{\prime} 39.09^{\prime \prime} \mathrm{N}$, 76¹6'8.7"E,5.vii.15, coll: J. Sengupta,2우우 Bhagshung, Kangra district, $1755 \mathrm{mt}$, 32॰14'23.1"N, 76¹9'41.3"E,5.vii.15, coll: J. Sengupta, $60^{{ }^{*}} \sigma^{\pi} 9$ 우 우 Palampur, Kangra district, $1220 \mathrm{mt}, 32^{\circ} 6^{\prime} 39.09^{\prime \prime N}, 76^{\circ} 16^{\prime} 8.7 " \mathrm{E}, 5 . v i i .15$, coll: J. Sengupta, $50^{x} 0^{x} 12$ 우 ㅇ Chabutar Village, Hamirpur district, $774 \mathrm{mt}, 31^{\circ} 41^{\prime} 10.2^{\prime \prime} \mathrm{N}$, 76³1'16.7"E, 2.ii.16, coll: J. Sengupta, 1 + Binjimandi, Mandi district, $1023 \mathrm{mt}$, 31'43'51.5"N, 7656'30.4"E, 27.iv.18, coll: J. Sengupta1 + Mandi, Mandi district, $1023 \mathrm{mt}$, 31'43'51.5"N, 7656'30.4"E, 24.iv.18, coll: J. Sengupta, 4 오 Sahoo River side, Mandi district,930 $\mathrm{mt}, \quad 32^{\circ} 33^{\prime} 12.1062 " \mathrm{~N}$, 7207'32.9088"E, 24.iv.18, coll: J. Sengupta, $50^{\pi} \sigma^{\pi}$ Nurchok, Mandi district, 784 $\mathrm{mt}, \quad 31^{\circ} 36^{\prime} 30.6792 " \mathrm{~N}, \quad 76^{\circ} 54^{\prime} 55.1808^{\prime \prime} \mathrm{E}$, 15.iv.18, coll: J. Sengupta, $50^{\pi} 0^{\pi}$ Bhagpur, Mandi district, $855 \mathrm{mt}, 31^{\circ} 17^{\prime} 37.6866^{\prime \prime} \mathrm{N}$, 7652'14.3574"E, 14.iv.18, coll: J. Sengupta, 4 우 우 $50^{\pi} 0^{\pi}$ Bergaon, Solan district, $1454 \mathrm{mt}, 30^{\circ} 55^{\prime} 14.2^{\prime \prime} \mathrm{N}, 77^{\circ} 06^{\prime} 18.1$ "E, 18.iv.17, coll: J. Sengupta, $30^{\pi} 0^{x}$ Khauoo, Shimla district, $1739 \mathrm{mt}, \quad 30^{\circ} 52^{\prime} 54.33^{\prime \prime} \mathrm{N}$, 77³8'17.49"E,, 20.iv.17, coll: J. Sengupta, $20^{\pi} 0^{\pi}$ Majher, Bilaspur district,1001 mt, 31'15'0.33"N, 76²9'54.74"E,4.ii.16,coll: J sengupta,2 o + Shishamati , Kullu district, 1248

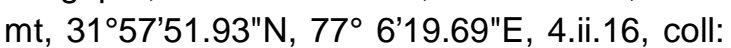
J. Sengupta, $3 \sigma^{\pi} \sigma^{\pi}$ Mohal ,Kullu district, 1205 $\mathrm{mt}, 31^{\circ} 54^{\prime} 56.73^{\prime \prime} \mathrm{N}, 7^{\circ} 7^{\prime} 1.588^{\prime \prime} \mathrm{E}$, 4.ii.16, coll: J. Sengupta, $30^{\pi} 0^{x}$ Sarvari, Kullu district, $1202 \mathrm{mt}$, 31'57’37.15"N, 776'47.23"E， 7.ii.16,: J. Sengupta, $10^{\top}$ Dhalpur, Kullu district, $1212 \mathrm{mt}$, 3157'16.37"N, $77^{\circ}$ 6’42.68"E,7.ii.16, coll: J. 
Sengupta, $20^{\pi} 0^{\pi}$ Gharakhar, Kullu district, 1436mt, 31'57'35.61"N, 77²'24.66"E, 7.ii.16, coll: J. Sengupta, 1 우 Renuka lake, Sirmour district, $672 \mathrm{mt}, 32^{\circ} 33^{\prime} 12.1^{\prime \prime} \mathrm{N}, 76^{\circ} 7^{\prime} 32.9^{\prime \prime} \mathrm{E}$, 15.iv.17, coll: J. Sengupta, $30^{\pi} 0^{x}$ Bergaon, Solan district, $1452 \mathrm{mt}, 30^{\circ} 54^{\prime} 16.1^{\prime \prime} \mathrm{N}$, 775'48.2"E, 18.iv.17, coll: J. Sengupta.

Distribution: India: Himachal Pradesh, Arunachal Pradesh, Jammu \& Kashmir, Manipur, Meghalaya, Mizoram, Nagaland, Sikkim, Uttarakhand, West Bengal.

Elsewhere: Australasian Region (Australia, New Zealand), Indo-Australian Region (Hawaii), Palaearctic Region (China, Japan), Oriental Region (Afghanistan, Myanmar, Pakistan, Sri Lanka).

XVIII. Genus Mallota Meigen, 1822

Subgenus Mallota Meigen, 1822

33. Mallota (Mallota) orientalis
Wiedemann, 1824 1842. Imatisma orientalis Macquart, Dipt. Exot. 2(2):69

Type-locality: Indonesia. Java.

Material examined: $20^{x} 0^{x}$ Bergaon, Solan district, $1452 \mathrm{mt}, 30^{\circ} 54^{\prime} 16.1^{\prime \prime N}, 77^{\circ} 5^{\prime} 48.2^{\prime \prime} \mathrm{E}$, 18.iv.17, coll: J. Sengupta, $30^{x} 0^{x} 1$ 아 kamthan Kalan, Solan district, $949 \mathrm{mt}, 30^{\circ} 55^{\prime} 58.53^{\prime \prime} \mathrm{N}$, $77^{\circ}$ 0’52.41"E, 18.iv.17, coll: J. Sengupta,

Distribution: India: Himachal Pradesh, Sikkim, West Bengal.

Elsewhere: Oriental region (Taiwan, Java, Laos)

XIX. Genus Mesembrius Rondani,
1857

Subgenus Mesembrius Rondani, 1857

34. Mesembrius

(Mesembrius) bengalensis (Wiedemann, 1819)

1819. Eristalis bengalensis Wiedemann, Zool. Mag. (Wied) 1: 16.

Type-locality: Bengal, India

Material examined: $10^{x}$ Renuka Lake, Sirmour district, 650mt, $32^{\circ} 33^{\prime} 12.1^{\prime \prime} \mathrm{N}$, 767'32.9"E, 15.iv.17, coll: J.Sengupta, 1 우 Renuka lake, Sirmour district, $672 \mathrm{mt}$,
32॰33'12.1"N,767'32.9"E, 15.iv.17, coll: J. Sengupta, 5 우,Bhanera, Hamirpur, Himachal Pradesh, India 953mt, 31³8'0.65"N, 76³9'43.42"E, 4.ii. 2016, coll: J. Sengupta, $30^{\pi} 0^{\pi}$ Batyana, Hamirpur, Himachal Pradesh,India, $784 \mathrm{mt}, \quad 31^{\circ} 29^{\prime} 4.49^{\prime \prime} \mathrm{N}$, 76³1'50.78"E, 4. ii.2016, 20 $0^{\pi} 0^{\pi}$ Karsai, Hamirpur, Himachal Pradesh, India $744 \mathrm{mt}$, $31^{\circ} 32^{\prime} 49.80 " \mathrm{~N}, 76^{\circ} 28^{\prime} 30.83 " \mathrm{E}, 2$.iv.2016

Distribution: India: Himachal Pradesh, West Bengal.

Elsewhere: Australasian Region (Australia, New Guinea)

XX. Genus Lycastris Walker, 1857

35. Lycastris flavohirta Brunetti, 1907

1907, Lycastris flavohirta Brunetti, 1907 Rec. Indian Mus.1: 379-380

Type-locality: India, West Bengal, Darjeeling

Material examined: $20^{x} \sigma^{x}$ Bahli, Shimla district, $1634 \quad \mathrm{mt}, \quad 31^{\circ} \quad 9^{\prime} 17.81^{\prime \prime} \mathrm{N}$, 7743'47.08"E,, 20.iv.17, coll: J. Sengupta.

Distribution: India: Himachal Pradesh, West Bengal

Elsewhere: Oriental Region (Nepal)

XXI. Genus Syritta Lepeletier \& Serville, 1828

36. Syritta indica (Wiedemann, 1824)

1884. Syritta rufifacies Bigot, Ann. Soc. Ent. Fr. Ser. 6, 3: 535-560.

=Syritta femorata Sack, 1913

=Syritta rufifacies Bigot, 1884

Type-locality: East Indies.

Material examined: 1 우 Sohari, Una district, $677 \mathrm{mt}, \quad 31^{\circ} 40^{\prime} 37.94 " \mathrm{~N}, \quad 7^{\circ} 18^{\prime} 0.91^{\prime \prime} \mathrm{E}$, 12.ii.16,coll: J. Sengupta.

Distribution: India: Himachal Pradesh, Assam, Bihar, Karnataka, Kerala, Madhya Pradesh, Maharashtra, Puducherry, Tamil Nadu, Uttarakhand, West Bengal.

Elsewhere: Oriental Region (Nepal, Sri Lanka)

37. Syritta pipiens (Linnaeus, 1758) 
1758. Musca pipiens Linnaeus, Syst.Nat. 10:594.

\section{Type-locality: Europe}

Material examined: 4 우 우 Sarahan, Kinnaur district, $1280 \mathrm{mt}, 31^{\circ} 30^{\prime} 53^{\prime \prime} \mathrm{N}, 7^{\circ} 44^{\prime} 56^{\prime \prime} \mathrm{E}$, 13.iv.18,coll: J.Sengupta, $20^{\pi} 0^{\pi}$, 1 우 Sangra, Kinnaur district, $1450 \mathrm{mt}, 31^{\circ} 33^{\prime} 16 " \mathrm{~N}, 77^{\circ} 55$ '7"E, 13.iv.18,coll: J. Sengupta, $20^{x} 0^{x}$ Bahli, Shimla district, $1634 \mathrm{mt}, 31^{\circ} 9^{\prime} 17.81^{\prime \prime} \mathrm{N}$,

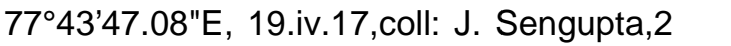
Palampur, Kangra district, $1220 \mathrm{mt}$, 326'39.09"N, 76¹6'8.7"E, 5.vii.15, coll: J. Sengupta, 1 ㅇ Renuka lake, Sirmour district, $672 \mathrm{mt}, 32^{\circ} 33^{\prime} 12.1 " \mathrm{~N}, 7^{\circ} 7^{\prime} 32.9 " \mathrm{E}, 15 . i v .17$, coll: J. Sengupta.

Distribution: India: Himachal Pradesh, Jammu \& Kashmir, Uttarakhand, Uttar Pradesh and West Bengal.

Elsewhere: Nearctic Region (California \& Florida), Neotropical region (British Columbia, Mexico), Oriental Region (Afghanistan, Nepal, Pakistan), Palearctic Region (USA)
XXII. Genus Xylota Meigen, 1822

Subgenus Xylota Meigen, 1822

38. Xylota (Xylota) nursei Brunetti, 1923

1923. Xylota nursei Brunetti.Fauna.Br. India; 3:240.

Type-locality: Shimla, India

Material examined: $10^{x}$ Khauoo, Shimla district, $1739 \mathrm{mt}, \quad 30^{\circ} 52^{\prime} 54.33^{\prime \prime} \mathrm{N}$, 77³8'17.49"E,,20.iv.17, coll: J. Sengupta,2우 우 Bergaon, Solan district, $1454 \mathrm{mt}, 30^{\circ} 55^{\prime} 14.2^{\prime \prime} \mathrm{N}$, 7706'18.1"E, 18.iv.17, coll: J. Sengupta,2우 우 Palampur, Kangra district, $1220 \mathrm{mt}$, 326'39.09"N, 76¹6’8.7"E, 5.vii.15, coll: J. Sengupta, $20^{\pi} 0^{\pi}$ Chabutar Village, Hamirpur district, $774 \mathrm{mt}, 31^{\circ} 41^{\prime} 10.2^{\prime \prime} \mathrm{N}, 76^{\circ} 31^{\prime} 16.7^{\prime \prime} \mathrm{E}$, 2.ii.16, coll: J. Sengupta.

Distribution: India: Himachal Pradesh, West Bengal.

Elsewhere: Nil.

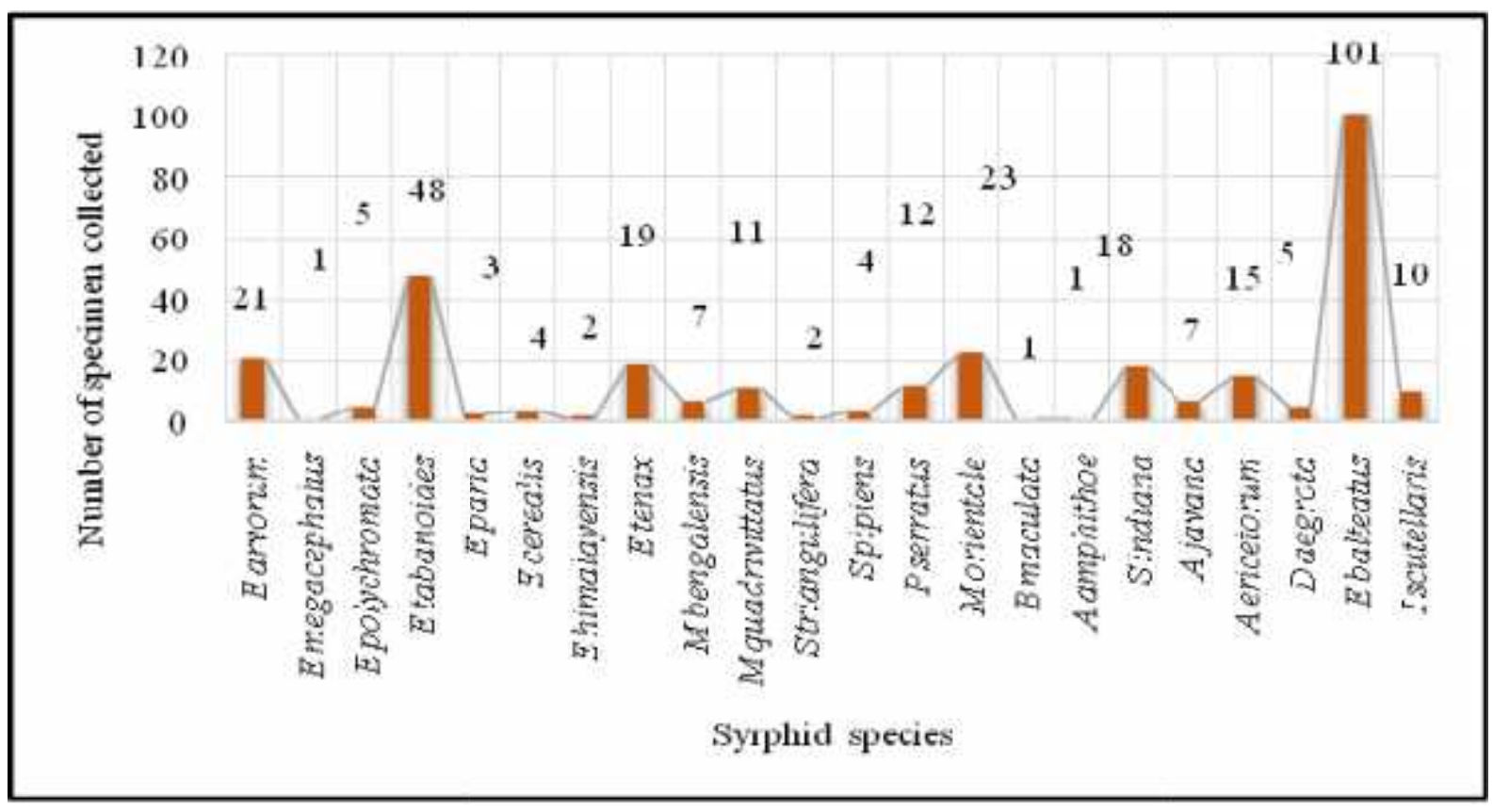

Figure 3: Abundance status of syrphid species from Mid hill Zone, Himachal Pradesh

\section{Discussion}

Altogether 38 species of hoverflies under 22 genera and 2 sub families have been reported from our study area. Among which 2 species namely Scavea pyrastri (Linnaeus, 1758) and Eristalinus (Eristalinus) polychromata
(Fabricius, 1787) has been reported from the first time from this mid hill zone as well as from the state of Himachal Pradesh. Elevation wise this zone represents the longest elevational span of the state. Therefore eventually the diversity of hoverflies are also quite high from 
this zone. Among the reported 38 species, 7 species found to be widely distributed throughout the year while 5 species found to be endemic from the state as well as from India. Such higher percentile of endemism represents the significance of this zone from biodiversity aspect. Further studies on pollinating hoverflies I at seasonal and periodic interval will give a clear cut scenario of the taxonomic aspects of these flies from this zone.

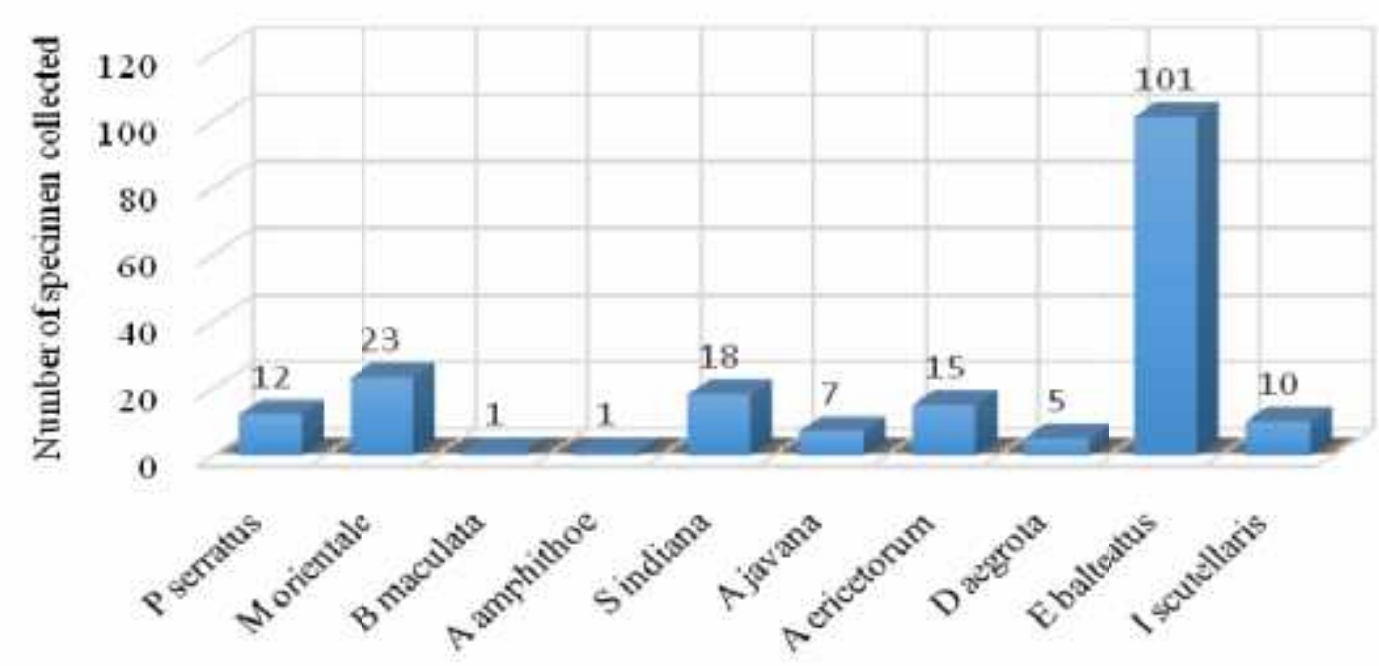

Syrphid species

Figure 4: Abundance status of species belonging to Syrphinae subfamily from Mid hill Zone, Himachal Pradesh 


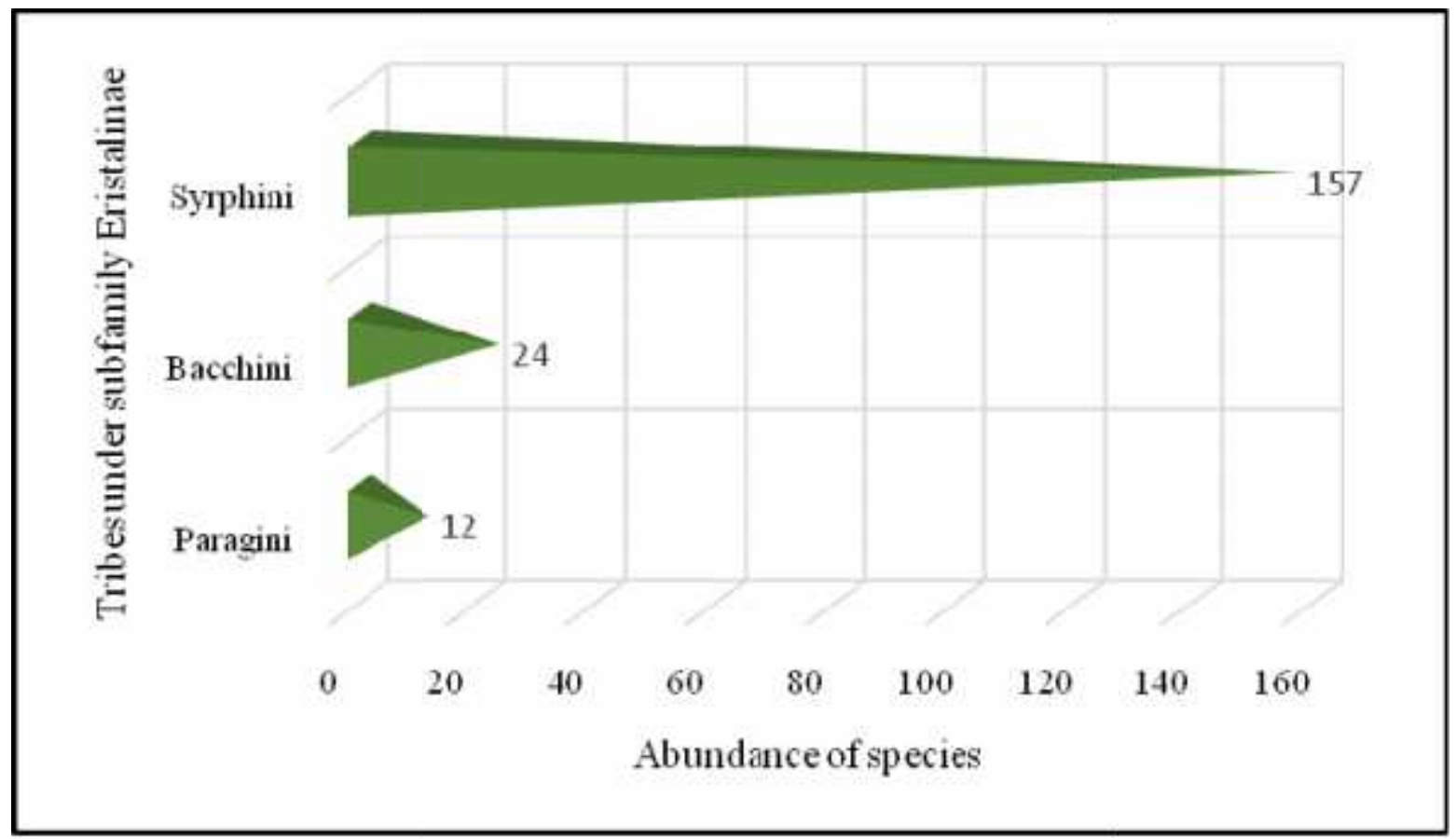

Figure 5: Abundance status of different tribes under Syrphinae subfamily from Mid hill zone, Himachal Pradesh

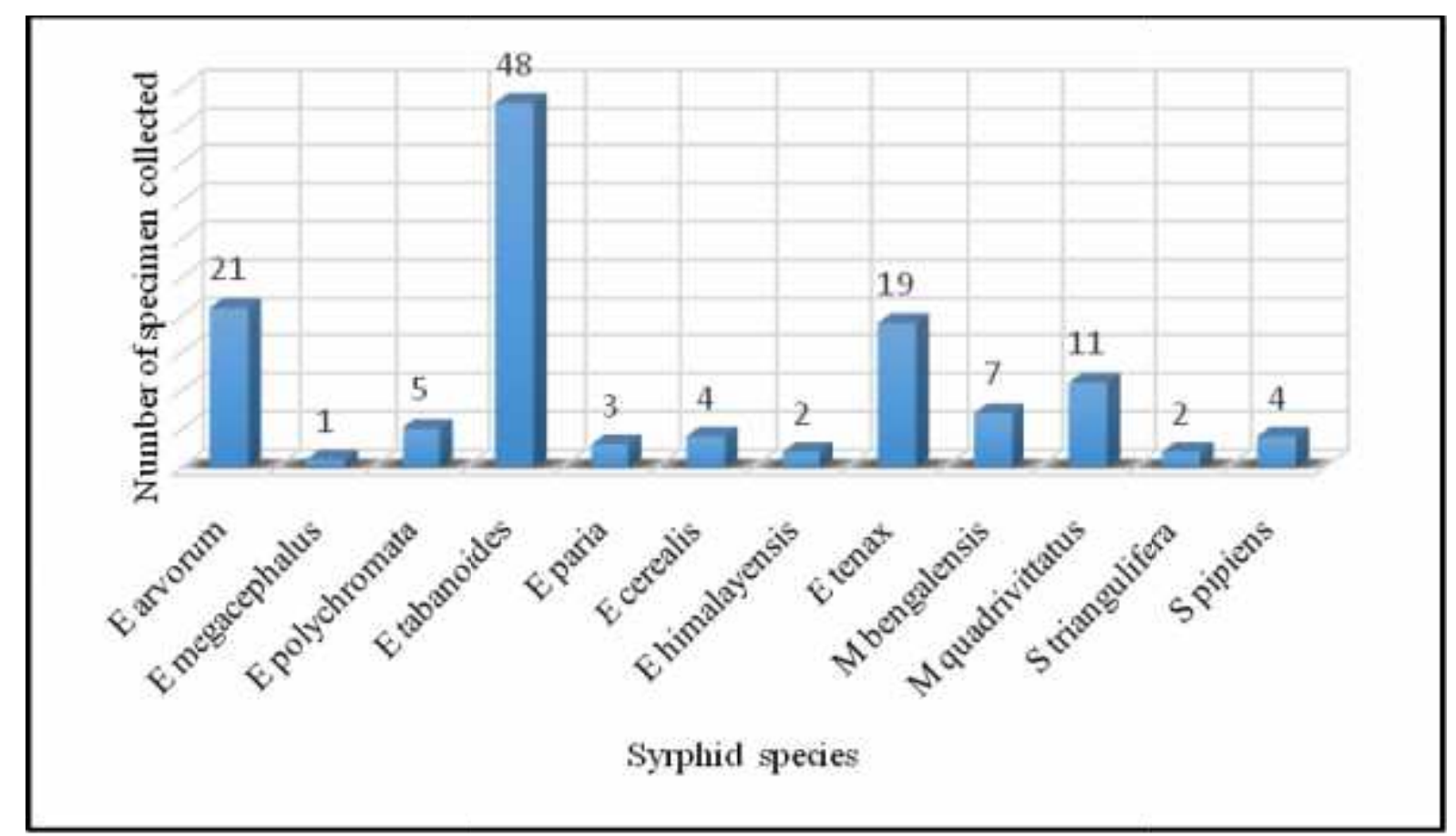

Figure 6: Abundance status of species belonging to Eristalinae subfamily from Mid hill Zone, Himachal Pradesh 


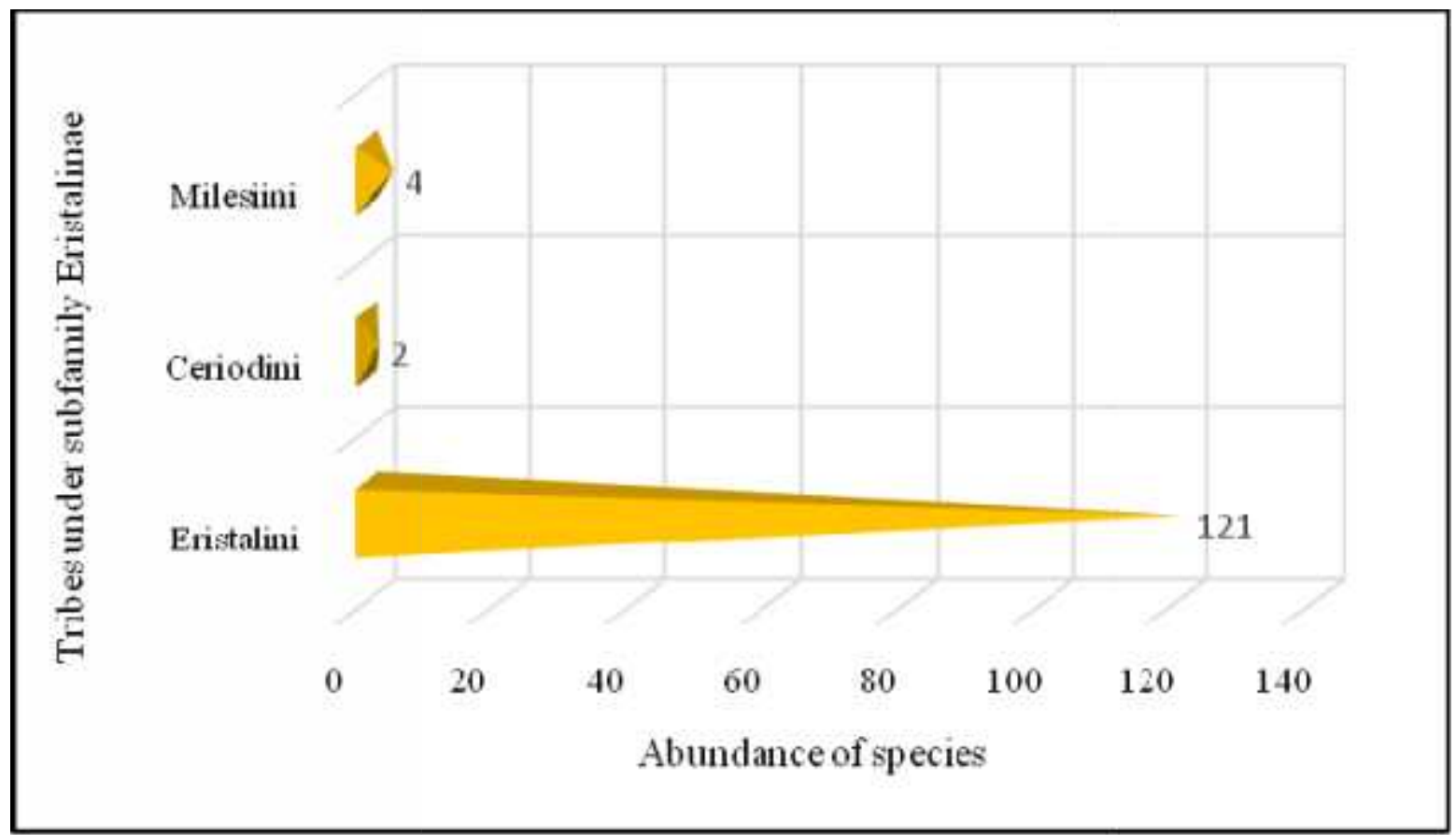

Figure 7: Abundance status of different tribes under Eristalinae subfamily from Mid hill Zone, Himachal Pradesh

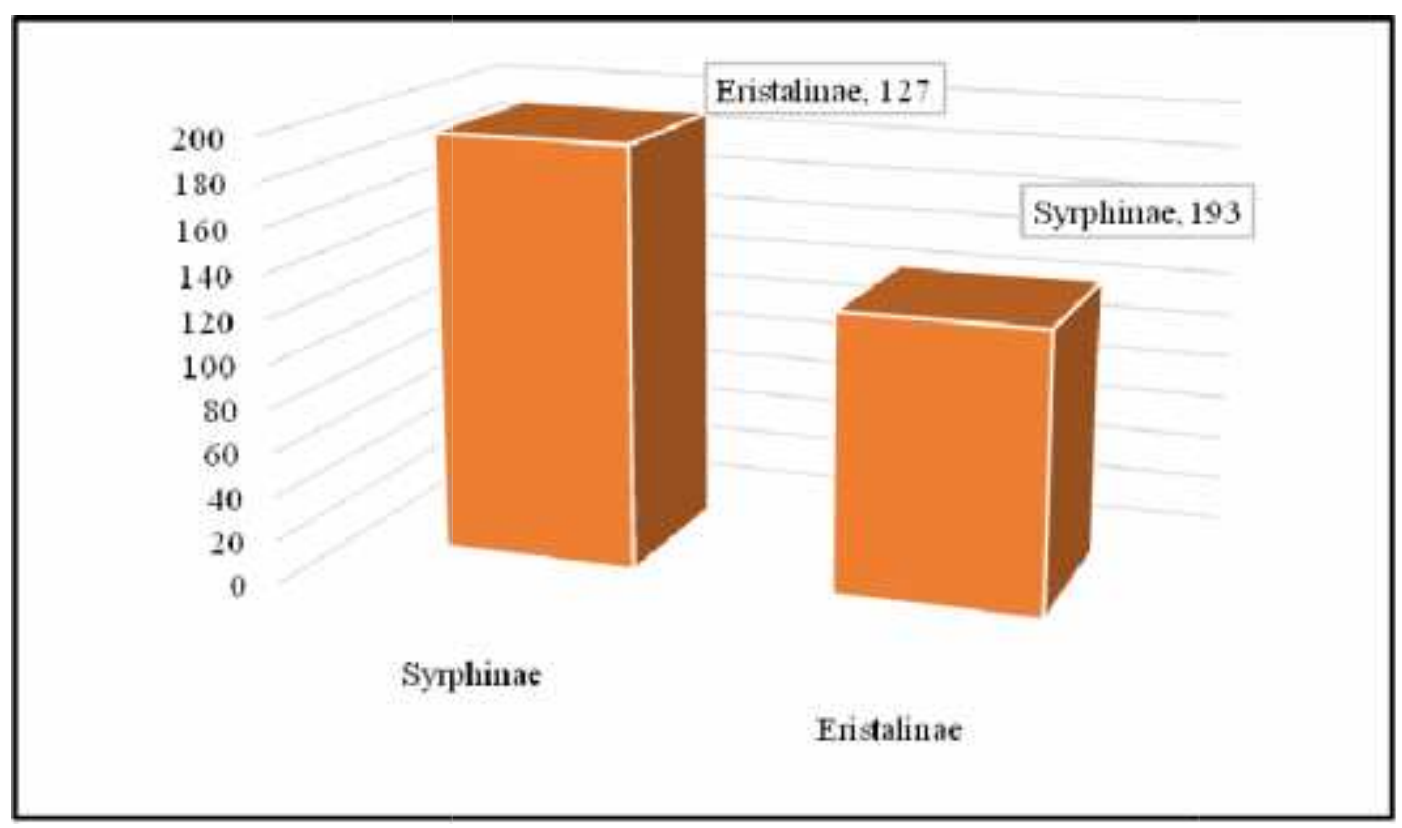

Figure 8: Comparative account of Abundance status of two subfamilies from Mid hill Zone, Himachal Pradesh

\section{Acknowledgments}

We wish to express our thanks to Dr. Kailash Chandra, Director, Zoological Survey of India for providing the permission and necessary facilities. Thanks are due to Dr. C. Raghunathan, Divisional. In Charge, 
Entomology. Division. B, for their continuous encouragement. Further, we acknowledge and convey our sincere thanks to our fellow team members for their constant encouragement.

\section{Conflicts of Interest}

\section{References}

Brunetti, E. (1907). Notes on the Oriental Syrphidae. Part I. Records of the Indian Museum, 1, 11-13.

Brunetti, E. (1908). Notes on Oriental Syrphidae with descriptions of new species. Part I. Records of the Indian Museum, 2, 49-96.

Brunetti, E. (1913). New and interesting Diptera from the eastern Himalayas. Records of the Indian Museum, 9(5), 255-277.

Brunetti, E. (1923). Family Syrphidae. Diptera Brachycera.. (Vol. 3). Taylor \& Francis.

Evenhuis, N.L. \& Pape, T. (editors). [2020]. Systema Dipterorum, Version [2.6]. http://diptera.org/, accessed on 02.02.2020.

Joshi, K., \& Bhatt, D. (2015). Avian species distribution along elevation at doon valley (foot hills of western Himalayas), Uttarakhand, and its association with vegetation structure. Journal of Asia-Pacific Biodiversity, 8(2): 158-167.

Lebuhn, G., Droege, S., Connor, E. F., Gemmill-Herren, B., Potts, S. G., Minckley, R. L., \& Cane, J. (2013). Detecting insect pollinator declines on regional and global scales. Conservation Biology, 27(1): 113-120.

Miranda, G. F. G., Young, A. D., Locke, M. M., Marshall, S. A., Skevington, J. H., \& Thompson, F. C. (2013). Key to the genera of Nearctic Syrphidae. Canadian Journal of Arthropod Identification, 23(1), 351. https://doi.org/10.3752/cjai.2013.23

Norman, C., DeCanio, S., \& Fan, L. (2008). The Montreal Protocol at 20: Ongoing opportunities for integration with climate protection. Global Environmental Change, 18(2):330-340.
The authors declare that the research was conducted in the absence of any commercial or economic associations that could be construed as a potential conflict of interest

Potts, S. G., Petanidou, T., Roberts, S., O'Toole, C., Hulbert, A., \& Willmer, P. (2006). Plant-pollinator biodiversity and pollination services in a complex Mediterranean landscape. Biological conservation, 129(4), 519-529.

https://doi.org/10.1016/j.biocon.2005.11.019

Ssymank, A., Kearns, C. A., Pape, T., \& Thompson, F. C. (2008). Pollinating flies (Diptera): a major contribution to plant diversity and agricultural production. Biodiversity, 9(1-2), 86-89. https://doi.org/10.1080/14888386.2008.9712892

Teskey, H.J., Vockeroth, J.R. \& Wood, D.M., (1981). Manual of Nearctic Diptera. Ottawa, Research Branch, Agriculture Canada, Monograph, 27.

Thompson, F. C., Vockeroth, J. R., \& Speight, M. C. (1982). The Linnaean species of flower flies (Diptera: Syrphidae).

Vockeroth, J. R. (1992). The flower flies of the subfamily Syrphinae of Canada, Alaska, and Greenland: Diptera, Syrphidae (Vol. 1867). Agriculture Canada. http://publications.gc.ca/pub?id=9.811395\&sl=0

Vockeroth, J. R., Thompson, F. C., McAlpine, J. F., Peterson, B. V., Shewell, G. E., Teskey, H. J., \& Wood, D. M. (1987). Syrphidae, pp. 713-743. Manual of Nearctic Diptera, 2. http://www.publications.gc.ca/pub?id=9.610315\&sl= $\underline{0}$ 